\title{
Formation of the Musca filament: evidence for asymmetries in the accretion flow due to a cloud-cloud collision ${ }^{\star}$
}

\author{
L. Bonne ${ }^{1}$, S. Bontemps ${ }^{1}$, N. Schneider ${ }^{2}$, S. D. Clarke ${ }^{2}$, D. Arzoumanian ${ }^{3}$, Y. Fukui ${ }^{4}$, K. Tachihara ${ }^{4}$, T. Csengeri ${ }^{5,1}$, \\ R. Guesten ${ }^{5}$, A. Ohama ${ }^{4}$, R. Okamoto ${ }^{4}$, R. Simon ${ }^{2}$, H. Yahia ${ }^{6}$, and H. Yamamoto ${ }^{4}$ \\ ${ }^{1}$ Laboratoire d'Astrophysique de Bordeaux, Université de Bordeaux, CNRS, B18N, allée Geoffrey Saint-Hilaire, 33615 Pessac, France \\ e-mail: lars. bonne@u-bordeaux. fr \\ 2 I. Physikalisches Institut, Universität zu Köln, Zülpicher Str. 77, 50937 Köln, Germany \\ ${ }^{3}$ Instituto de Astrofisica e Ciencias do Espaco, Universidade do Porto, CAUP, Rua das Estrelas, 4150-762 Porto, Portugal \\ ${ }^{4}$ Department of Physics, Nagoya University, Chikusa-ku, Nagoya 464-8602, Japan \\ ${ }^{5}$ Max-Planck-Institut für Radioastronomie, Auf dem Hügel 69, 53121 Bonn, Germany \\ ${ }^{6}$ INRIA Bordeaux Sud-Ouest, 33405 Talence, France
}

Received 28 April 2020 / Accepted 9 October 2020

\begin{abstract}
Context. Dense molecular filaments are ubiquituous in the interstellar medium, yet their internal physical conditions and the role of gravity, turbulence, the magnetic field, radiation, and the ambient cloud during their evolution remain debated.

Aims. We study the kinematics and physical conditions in the Musca filament, the ambient cloud, and the Chamaeleon-Musca complex to constrain the physics of filament formation.

Methods. We produced $\mathrm{CO}(2-1)$ isotopologue maps with the APEX telescope that cut through the Musca filament. We further study a NANTEN2 ${ }^{12} \mathrm{CO}(1-0)$ map of the full Musca cloud, H I emission of the Chamaeleon-Musca complex, a Planck polarisation map, line radiative transfer models, Gaia data, and synthetic observations from filament formation simulations.

Results. The Musca cloud, with a size of $\sim 3-6$ pc, contains multiple velocity components. Radiative transfer modelling of the CO emission indicates that the Musca filament consists of a cold $(\sim 10 \mathrm{~K})$, dense $\left(n_{\mathrm{H}_{2}} \sim 10^{4} \mathrm{~cm}^{-3}\right)$ crest, which is best described with a cylindrical geometry. Connected to the crest, a separate gas component at $T \sim 15 \mathrm{~K}$ and $n_{\mathrm{H}_{2}} \sim 10^{3} \mathrm{~cm}^{-3}$ is found, the so-called strands. The velocity-coherent filament crest has an organised transverse velocity gradient that is linked to the kinematics of the nearby ambient cloud. This velocity gradient has an angle $\geq 30^{\circ}$ with respect to the local magnetic field orientation derived from Planck, and the magnitude of the velocity gradient is similar to the transonic linewidth of the filament crest. Studying the large scale kinematics, we find coherence of the asymmetric kinematics from the $50 \mathrm{pc} \mathrm{H}$ i cloud down to the Musca filament. We also report a strong $\left[\mathrm{C}^{18} \mathrm{O}\right] /\left[{ }^{13} \mathrm{CO}\right]$ abundance drop by an order of magnitude from the filament crest to the strands over a distance $<0.2 \mathrm{pc}$ in a weak ambient far-ultraviolet (FUV) field.

Conclusions. The dense Musca filament crest is a long-lived (several crossing times), dynamic structure that can form stars in the near future because of continuous mass accretion replenishing the filament. This mass accretion on the filament appears to be triggered by a Hi cloud-cloud collision, which bends the magnetic field around dense filaments. This bending of the magnetic field is then responsible for the observed asymmetric accretion scenario of the Musca filament, which is, for instance, seen as a V-shape in the position-velocity (PV) diagram.
\end{abstract}

Key words. ISM: structure - ISM: kinematics and dynamics - ISM: individual objects: Musca - stars: formation - evolution

\section{Introduction}

The complexity of the interstellar medium (ISM) has been revealed in numerous continuum and molecular line studies, but it is only since the unprecedented far-infrared sensitivity of the Herschel Space Telescope (Pilbratt et al. 2010) that the ubiquity of dense filamentary structures in the ISM has been revealed and their integral role in the star formation process has been established. It was shown that almost all pre-stellar and protostellar cores are located in filaments or at a filament junction (e.g. André et al. 2010, 2014; Molinari et al. 2010; Bontemps et al. 2010; Könyves et al. 2010, 2015; Arzoumanian et al. 2011; Hill et al. 2011; Schneider et al. 2012; Rygl et al. 2013; Polychroni et al. 2013; Marsh et al. 2016). Spectral line observations of

\footnotetext{
* The reduced datacubes are only available at the CDS via anonymous ftp to cdsarc.u-strasbg.fr (130.79.128.5) or via http://cdsarc.u-strasbg.fr/viz-bin/cat/J/A+A/644/A27
}

filamentary structures have shown that many dust continuum filaments contain several velocity-coherent sub-filaments, the so-called fibers (e.g. Hacar et al. 2013; Tafalla \& Hacar 2015; Dhabal et al. 2018). However, recently some theoretical studies have indicated that coherent structures in velocity space are not necessarily coherent in three-dimensional space (Zamora-Avilés et al. 2017; Clarke et al. 2018). Massive filamentary structures in more distant regions, such as massive ridges (e.g. Schneider et al. 2010; Hennemann et al. 2012) and hub-filament systems (e.g. Myers 2009; Schneider et al. 2012; Peretto et al. 2013, 2014; Henshaw et al. 2017; Williams et al. 2018), are proposed to be the dominant way to form rich clusters of stars (e.g. Motte et al. 2018), and thus the bulk of star formation in our galaxy. It is therefore critical to unveil the precise physical processes at work to explain the formation of filaments of all types.

While early theoretical studies described filaments as structures confined by isotropic pressure equilibrium (Ostriker 1964; 
Inutsuka \& Miyama 1992), simulations support the argument that filaments are a manifestation of structure development caused by the thermodynamic evolution of the ISM during molecular cloud formation. All (magneto)-hydrodynamic ISM simulations with turbulence, including and not including selfgravity and/or a magnetic field, naturally produce filaments (e.g. Audit \& Hennebelle 2005; Heitsch et al. 2005; Hennebelle et al. 2008; Nakamura \& Li 2008; Banerjee et al. 2009; Gómez \& Vázquez-Semadeni 2014; Smith et al. 2014, 2016; Chen \& Ostriker 2014; Seifried \& Walch 2015; Federrath 2016; DuarteCabral \& Dobbs 2017). In these simulations, filaments are argued to originate from the collision of shocked sheets in turbulent flows (Padoan et al. 2001), by instabilities in self-gravitating sheets (Nagai et al. 1998), or as the result of long-lived coherent flows in the turbulent ISM (Hennebelle 2013) which could be at least partly convergent. The magnetic field can only reinforce the presence of filaments, as it increases local axisymetries and as it may stabilise and drive turbulent flows aligned with the field (Hennebelle 2013). The case of magnetised H I colliding streams and cloud-cloud collisions (e.g. Ballesteros-Paredes et al. 1999; Koyama \& Inutsuka 2002) is particularly interesting in this context as it naturally creates a significant level of turbulence and flows. It can also lead to bending of the magnetic field around pre-existing structures, which could then drive local convergent flows perpendicular to the dense filamentary structures (Inoue $\&$ Fukui 2013; Inoue et al. 2018). Such local convergent flows were indeed observed for massive dense cores by Csengeri et al. (2011), for instance. From an observational point of view, cloudcloud collisions at a velocity of $10-20 \mathrm{~km} \mathrm{~s}^{-1}$ have been argued to form massive star-forming filaments (Fukui et al. 2020, and references therein).

Any scenario to form filaments has to account for the most critical properties of observed filaments. The so-called universal (Arzoumanian et al. 2011, 2019; Koch \& Rosolowsky 2015), yet highly debated (e.g. Panopoulou et al. 2017; Seifried et al. 2017; Ossenkopf-Okada \& Stepanov 2019), filament width of $0.1 \mathrm{pc}$ in nearby molecular clouds is close to the sonic scale which could fit in a scenario where filaments are made of dense, post-shock gas of converging flows (Arzoumanian et al. 2011; Schneider et al. 2011; Federrath 2016). In the companion paper (Bonne et al. 2020), observational indications were found of warm gas from low-velocity shocks associated with mass accretion on the Musca filament. It has also led to some theoretical models considering gravitational inflow that might provide an explanation for this universal width (Heitsch 2013; Hennebelle \& André 2013). Indications of an inflowing mass reservoir as a result of gravity were presented in Palmeirim et al. (2013) and Shimajiri et al. (2019). In the medium surrounding dense filaments, striations are often found which are well aligned with the magnetic field (Goldsmith et al. 2008; Palmeirim et al. 2013; Alves de Oliveira et al. 2014; Cox et al. 2016; Malinen et al. 2016). This has often led to the interpretation of gas streaming along the magnetic field lines. So far, there is no strong observational evidence for this and recently it was argued from a theoretical study that striations might result from MHD waves (Tritsis \& Tassis 2016) in large pc-scale sheets. Similarly, the so-called fibers, seen as sub-structures of filaments, are found at different velocities as if they would originate from slightly different velocity flows inside the same global convergence of flows in the cloud. Several numerical simulations also observe fibers (e.g. Smith et al. 2014, 2016; Moeckel \& Burkert 2015; Clarke et al. 2017; Zamora-Avilés et al. 2017), while in pressure equilibrium models filaments naturally tend to fragment rapidly into cores (Inutsuka \& Miyama 1997).
In summary, these different views on the relation between filaments and the surrounding ambient cloud, in particular the understanding of the physical origin of star-forming filaments, require detailed observational studies and comparison with simulations. In this paper, we study the Musca filament which is probably at an early evolutionary state as its ambient cloud is not yet perturbed by star formation.

The following section introduces the Musca filament and why this filament is particulary interesting to study the relation with its ambient cloud. Section 3 describes the observations carried out with the APEX telescope and Sect. 4 presents the first results of the APEX and NANTEN2 observations. Section 5 provides a radiative transfer analysis of the observational data to constrain the physical conditions in the filament. In Sect. 6 the implications of these results on the formation of the Musca filament and its relation with the ambient cloud are discussed.

\section{The Musca filament and Chamaeleon clouds}

\subsection{The Musca filament}

We focus on the Musca filament which is seen on the sky as a 6 pc long filamentary structure in continuum, extinction and molecular lines (e.g. Mizuno et al. 2001; Kainulainen et al. 2009; Schneider et al. 2011; Hacar et al. 2016; Cox et al. 2016). This filament is located in the Chamaeleon-Musca complex, see Fig. 1, which is considered a single molecular complex with a size of $\sim 70 \mathrm{pc}$ on the plane of the sky if one includes Cham East (e.g. Corradi et al. 1997; Mizuno et al. 2001; Planck Collaboration Int XXVIII 2015; Liszt et al. 2019). Figure 2 presents the column density map of the Musca filament combining 2MASS and Herschel data which shows that this high column density filament with its ambient cloud is in relative isolation in the plane of the sky. The Musca filament hosts one protostellar core (VilasBoas et al. 1994; Juvela et al. 2012; Machaieie et al. 2017), and may contain a few prestellar cores (Kainulainen et al. 2016) with an average core separation that could fit with gravitational fragmentation inside a filamentary crest. The filament is thus likely at a relatively early evolutionary stage and has indeed a relatively low line mass compared to other more active star forming filaments like B211/3 in Taurus (Palmeirim et al. 2013; Cox et al. 2016; Kainulainen et al. 2016). As there is only one protostar which is located in the far north of the filament, the cloud is still unperturbed by protostellar feedback and is thus a very interesting location to study the formation of star forming gas and the role of the ambient cloud. The presence and structure of this ambient cloud was clearly established with high sensitivity dust continuum observations with Herschel and Planck (Cox et al. 2016; Planck Collaboration Int. XIII 2016), and will be described in more detail below.

For clarity in this paper, we distinguish four features of the Musca cloud based on the column density map and profile of dust emission, mostly following the nomenclature introduced by Cox et al. (2016).

Firstly, the (filament) crest is the high column density spine of the large filament in the Musca cloud with $N_{\mathrm{H}_{2}}>3 \times$ $10^{21} \mathrm{~cm}^{-2}$ (Cox et al. 2016), see Fig. 3. From fitting a Plummer profile to the average column density profile of the full Musca filament, working with a distance of $140 \mathrm{pc}$, this suggest a radius of $0.056 \mathrm{pc}$ for the filament crest (Cox et al. 2016).

Secondly, strands were defined in Cox et al. (2016) as the immediate surrounding of the filament crest where dust column density was found to be significant $\left(N_{\mathrm{H}_{2}} \sim 2 \times 10^{21} \mathrm{~cm}^{-2}\right)$ and inhomogeneous. For instance, there is a strong tendency to be 


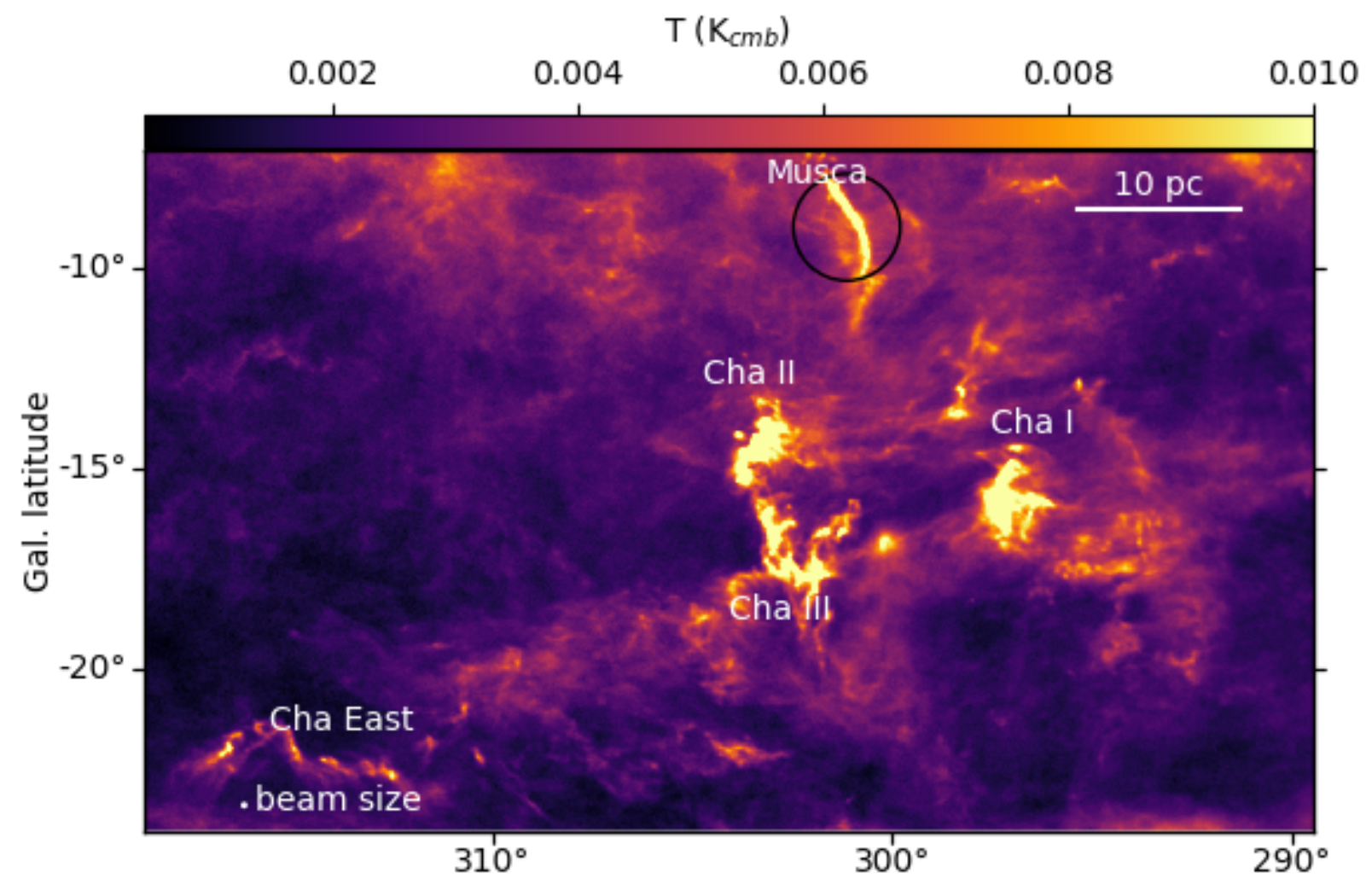

Gal. longitude

Fig. 1. Planck emission map at $353 \mathrm{GHz}$ of the Chamealeon-Musca complex (Planck Collaboration I 2016). The names of the relatively dense regions in the complex are indicated in white. The black circle indicates the region where Gaia data were extracted to investigate the distance of the Musca filament and cloud. The Planck resolution at $353 \mathrm{GHz}$ is $4.8^{\prime}$, which corresponds to a physical size of $0.25 \mathrm{pc}$ at a distance of $180 \mathrm{pc}$ (Zucker et al. 2019).

asymmetric with a brighter emission directly east of the filament crest, and the strands display a hair-like structure that is preferentially perpendicular to the filament crest (Cox et al. 2016), see Fig. 4. To go one step further we here propose that the strands could represent all column density in excess in Fig. 3 to the average Plummer profile fitted on the column density towards the crest. These strands might then contain the most nearby ambient gas of the filament crest and may represent a mass reservoir to be collected soon by the crest. In Fig. 3 and local cuts perpendicular to the filament axis, we found that the strands typically extend up to a distance of $\sim 0.4$ pc from the filament crest. It is not straightforward to fit a function to the strands, but from Fig. 3 it can be observed that the column density excess to the Plummer fit typically extends up to a distance of $0.4 \mathrm{pc}$. The presence of the strands leads to an asymmetric column density profile for Musca. Other asymmetric column density profiles were already observed in the Pipe Nebula (Peretto et al. 2012).

The ambient Musca cloud then embeds the filament crest and strands, and has a typical average column density of $N_{\mathrm{H}_{2}} \sim 0.8 \times 10^{21} \mathrm{~cm}^{-2}$ within a typical distance of $3 \mathrm{pc}$ from the filament crest, see Figs. 2 and 3.

Lastly, Striations are filamentary structures in the parsec scale ambient cloud and sub-parsec scale strands of the Musca filament, see Fig. 4. The striations are well aligned with the magnetic field on the plane of the sky (Cox et al. 2016). This magnetic field is roughly perpendicular to the Musca filament crest, as is also found for other dense filamentary structures (Planck Collaboration Int. XXXV 2016; Planck Collaboration Int. XIII 2016) and even at the centre of massive ridges like
DR21 (Vallée \& Fiege 2006; Schneider et al. 2010). Using $\mathrm{C}^{18} \mathrm{O}$ observations it was proposed that the filament crest is a velocitycoherent structure (Hacar et al. 2016), or a single fiber following the nomenclature of Hacar et al. (2013), unlike well studied filaments such as B211/3 in Taurus and Serpens south (Hacar et al. 2013; Dhabal et al. 2018). This makes the Musca filament relatively simple in velocity space.

More recently, it was questioned whether the Musca filament can be described as truely cylindrical (in constrast to a sheet seen edge-on) by proposing that the striations pattern is reflecting magnetohydrodynamic vibrations which would require a large, pc-scale region of emission in contradiction to the $0.1 \mathrm{pc}$ width of the crest. It would then suggest that the Musca cloud is a sheet that is seen edge-on and thus only appears filamentary due to projection (Tritsis \& Tassis 2018). We use our new observations and an updated view of the global structure of the cloud to rediscuss this important issue. Meanwhile we continue to use the term "filament", at least for the crest, throughout the paper.

\subsection{Distance of the Musca filament}

In earlier studies, the distance of the Musca cloud was generally estimated to be 140-150 pc (e.g. Franco 1991; Knude \& Hog 1998). Studying the reddening of stars in the Gaia DR2 data release (Gaia Collaboration 2018; Andrae et al. 2018) that are close to the Musca filament, with a method similar to Yan et al. (2019), shows indeed a noteworthy reddening increase at a distance of 140-150 pc, see Fig. 5. The region in the plane of the 


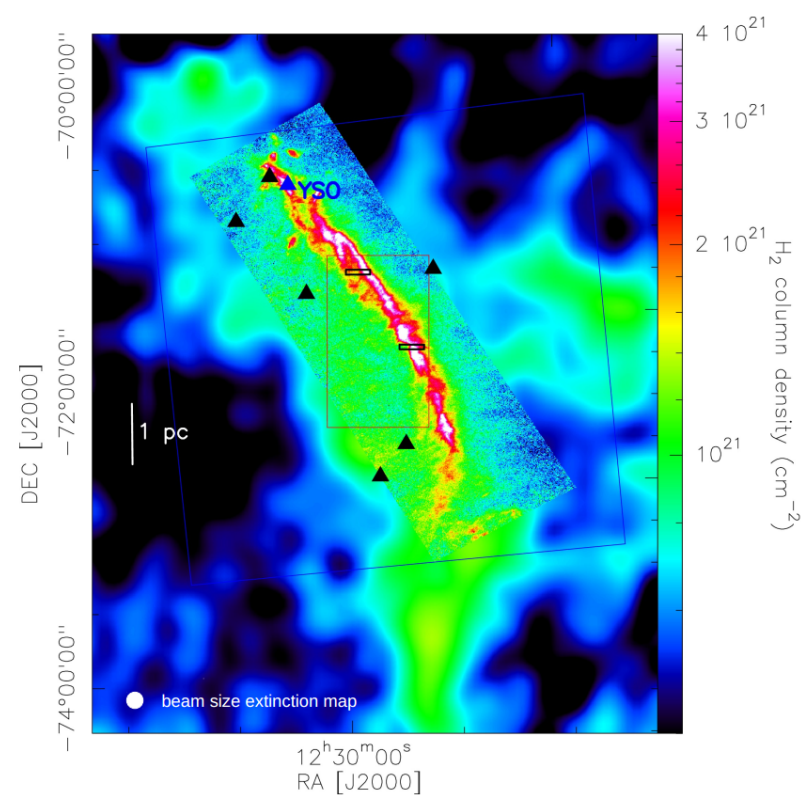

Fig. 2. Column density map of the Musca filament from the HGBS (André et al. 2010; Cox et al. 2016) embedded in the large scale 2MASS extinction map of Musca, scaled to the Herschel column density, that traces the ambient cloud. The extinction map was produced by the $A_{\mathrm{V}}$ mapping tool in Schneider et al. (2011). The black boxes indicate the maps made with the APEX telescope, the red box indicates the area displayed in Fig. 4, and the blue box indicates the area mapped with the NANTEN2 telescope. The black triangles show the locations of the stars with a distance smaller than $140 \mathrm{pc}$ and a significant reddening $(>0.3)$ in the Gaia catalogue. The blue triangle shows the location of the only young stellar object (YSO) in the Musca filament.

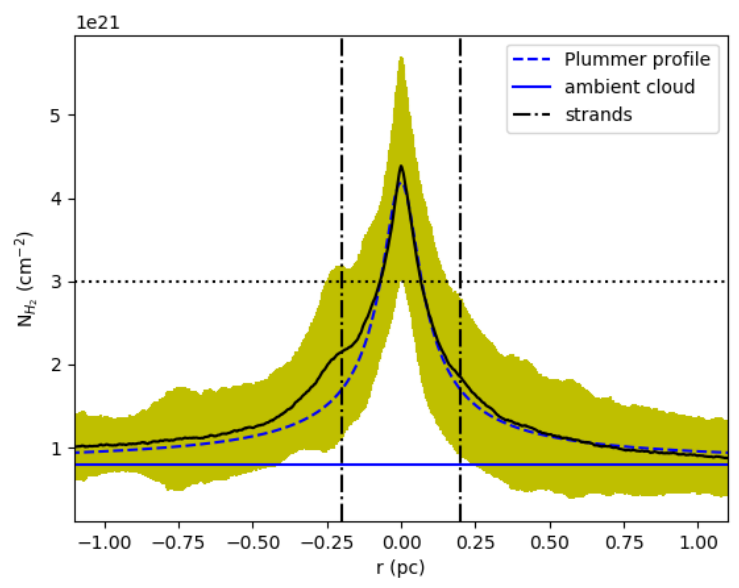

Fig. 3. Average column density profile of the Musca filament from the Herschel data (Cox et al. 2016), corrected to a distance of 140 pc. The blue horizontal line indicates the column density associated with the ambient cloud $\left(N_{\mathrm{H}_{2}} \sim 0.8 \times 10^{21} \mathrm{~cm}^{-2}\right)$. The dashed blue line shows the fitted Plummer profile to the column density (excluding the strands). The vertical lines indicate the location of column density excess with respect to the Plummer fit close to the filament crest, the so-called strands. The dashed horizontal black line indicates $N_{\mathrm{H}_{2}}=3 \times 10^{21} \mathrm{~cm}^{-2}$, which is the minimal column density used to define the filament crest.

sky studied with Gaia data is displayed in Fig. 1. For more information on the determination of the distance, see Appendix A. It can however be noted in Fig. 5 that there is already reddening for a couple of stars starting at $100 \mathrm{pc}$ towards the Musca cloud. One should note that there are significant uncertainties on the reddening in the Gaia catalogue, but this behaviour can also

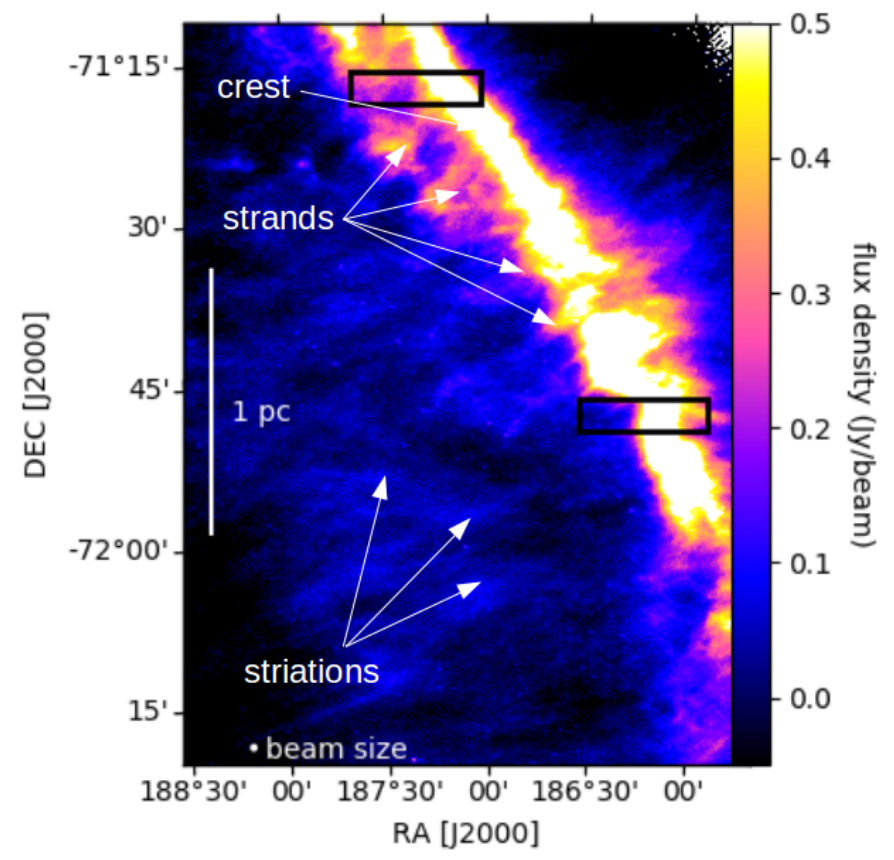

Fig. 4. Zoom (red box in Fig. 2) in the Herschel $250 \mu \mathrm{m}$ map of the Musca cloud (Cox et al. 2016). The hair like structures perpendicular to the Musca filament, in the strands and ambient cloud, are indicated in white. The black boxes indicate the regions covered by the APEX maps.

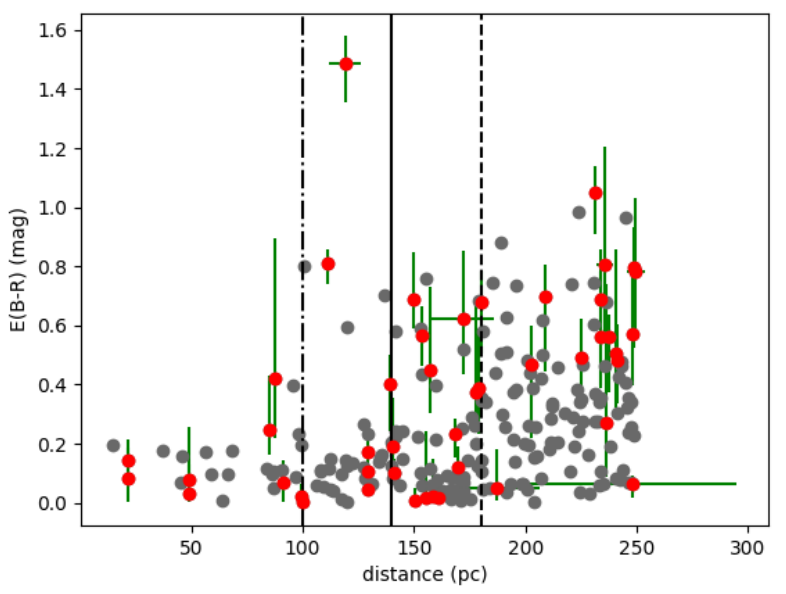

Fig. 5. Reddening obtained with the Gaia telescope as a function of distance for stars in the region of the Musca cloud. The coloured points are observed at locations where the Planck $353 \mathrm{GHz}$ emission is above the threshold that was used to define the area covered by the Musca cloud, see Appendix A. This shows a general increase of the reddening at a distance of $140 \mathrm{pc}$, shown with the full vertical line, but there also appears to be some reddening starting at $90-100 \mathrm{pc}$, indicated with the dashdot vertical line. The dashed vertical line indicates the distance of the Chamaeleon I, II and III molecular clouds at $183 \mathrm{pc}$ from Zucker et al. (2019).

be noted for two stars in Franco (1991). In the Gaia catalogue, the location of the stars with significant reddening at a distance $<140 \mathrm{pc}$ is spread over the full Musca cloud, see Fig. 2. This points to the fact that there might be some extended nearby gas at a distance of $\sim 100 \mathrm{pc}$ towards the Musca region. Since the Chamaeleon-Musca complex has a size of $\sim 70 \mathrm{pc}$ in the plane of the sky, it is not unlikely that the cloud has a similar size along the line of sight. This could possibly lead to extinction starting at $100 \mathrm{pc}$ for some stars. However, the clearest jump in reddening 
happens around $140 \mathrm{pc}$, which is consistent with several earlier studies (Franco 1991; Corradi et al. 1997; Whittet et al. 1997; Knude \& Hog 1998) of the high column density Musca cloud. We thus assume a distance of $140 \mathrm{pc}$ for the Musca filament in this paper. Note that this distance is smaller than the distance of $183 \pm 3 \pm 9$ pc derived for the dense Chamaeleon clouds (Cha I, Cha II and Cha III) in Zucker et al. (2019). Inspecting the reddening as a function of distance with the Gaia data, we also found a distance of 180-190 pc for these dense Chamaeleon clouds, see Appendix A. This would indicate that Musca has a slightly more nearby location than the Chamaeleon clouds.

\section{Observations}

\subsection{APEX: PI230 observations}

In September 2018, we performed observations with the PI230 receiver which is installed on the APEX telescope (Güsten et al. 2006). The two bands of PI230, each with $\sim 8 \mathrm{GHz}$ bandwidth, were tuned to a frequency to cover $213.7-221.4 \mathrm{GHz}$ and 229.5 237.2 GHz such that the ${ }^{12} \mathrm{CO}(2-1),{ }^{13} \mathrm{CO}(2-1), \mathrm{C}^{18} \mathrm{O}(2-1)$ and $\mathrm{SiO}(5-4)$ lines, respectively at 230.538, 220.399, 219.560 and 217.105 GHz, were observed simultaneously. The observations with PI230 were performed in the on-the-fly mode, creating two maps in all these lines with a size of $600^{\prime \prime} \times 100^{\prime \prime}$. These two maps cover the Musca filament crest and its nearby strands in two different regions, see Fig. 2. The northern map of the two is located towards a region that has a filamentary shape and the strands to the east, while the southern map is located in a region that shows signs of fragmentation with strands to the west. The OFF position used for the observations is located at $\alpha_{(2000)}=$ $12^{\mathrm{h}} 41^{\mathrm{m}} 38^{\mathrm{s}} ; \delta_{(2000)}=-71^{\circ} 11^{\prime} 00^{\prime \prime}$, identical to the OFF position used for the observations presented in Hacar et al. (2016). This OFF position was checked using another OFF position that is located further away from the Musca filament, at $\alpha_{(2000)}=$ $12^{\mathrm{h}} 42^{\mathrm{m}} 21^{\mathrm{s}} ; \delta_{(2000)}=-72^{\circ} 27^{\prime} 31^{\prime \prime}$, which was selected based on Planck maps. We found no contamination for any of the lines, for example, for ${ }^{12} \mathrm{CO}(2-1)$ the baseline rms was $2.8 \times 10^{-2} \mathrm{~K}$.

The spectral resolution of these observations is $\sim 0.08 \mathrm{~km} \mathrm{~s}^{-1}$, which resolves the velocity-coherent filament crest component with $\sigma \sim 0.15 \mathrm{~km} \mathrm{~s}^{-1}$ presented in Hacar et al. (2016). The spatial resolution of the observations is $\sim 28^{\prime \prime}$, and inside a velocity interval of $0.1 \mathrm{~km} \mathrm{~s}^{-1}$, the typical rms is $\sim 0.07 \mathrm{~K}$. The main beam efficiency is $\eta_{\mathrm{mb}}=0.68^{1}$, and the forward efficiency used is 0.95 . The data reduction was performed using the CLASS software ${ }^{2}$.

\subsection{APEX: FLASH+ observations}

With $\mathrm{FLASH}^{+}$(Klein et al. 2014) on the APEX telescope, the ${ }^{12} \mathrm{CO}(3-2),{ }^{12} \mathrm{CO}(4-3)$ and ${ }^{13} \mathrm{CO}(3-2)$ lines at 345.796, 461.041 and $330.588 \mathrm{GHz}$, respectively were observed simultaneously. $\mathrm{FLASH}^{+}$consists out of two receivers that can observe simultaneously: FLASH345 and FLASH460. Both receivers have two bands with a $4 \mathrm{GHz}$ bandwidth. FLASH345 can observe in the 268-374 GHz range, while FLASH460 can observe in the 374$516 \mathrm{GHz}$ range. These observations were performed towards the northern and southern map. Towards the northern area, the $\mathrm{FLASH}^{+}$observations make a map of $500^{\prime \prime} \times 100^{\prime \prime}$ that covers an area including the filament crest and the eastern strands, and in the southern area the $\mathrm{FLASH}^{+}$map only covers an area of $120^{\prime \prime} \times 100^{\prime \prime}$ which is centered on the filament crest. The

1 http://www .apex-telescope.org/telescope/efficiency/

2 http://www.iram. fr/IRAMFR/GILDAS observations towards the southern map were performed in a setup that did not cover the ${ }^{13} \mathrm{CO}(3-2)$ line. As a result this line is only available for the northern map. These observations with $\mathrm{FLASH}^{+}$were spread over 3 observing periods: July 2017 (P100), from May to June 2018 (P101) and September 2018 (P102). All observations experienced certain complications requiring specific attention. The $\mathrm{P} 100{ }^{12} \mathrm{CO}(3-2)$ observations have some contamination from the used OFF position (at $\left.\alpha_{(2000)}=12^{\mathrm{h}} 24^{\mathrm{m}} 05^{\mathrm{s}} ; \delta_{(2000)}=-71^{\circ} 23^{\prime} 45^{\prime \prime}\right)$. For ${ }^{12} \mathrm{CO}(3-2)$ a correction for the contamination from the OFF position was carried out by fitting a gaussian to the contamination in the OFF position and adding it to all spectra observed with this OFF position. This gaussian has $T_{\mathrm{A}}^{*}=1.17 \mathrm{~K}, v=$ $3.05 \mathrm{~km} \mathrm{~s}^{-1}$ and a $F W H M=0.418 \mathrm{~km} \mathrm{~s}^{-1}$. For the ${ }^{12} \mathrm{CO}(4-3)$ observations, no contamination was found in this OFF position at a baseline rms of $0.09 \mathrm{~K}$ within $0.1 \mathrm{~km} \mathrm{~s}^{-1}$. In P101, both the observations with FLASH345 and the FLASH460 instruments were shifted by respectively -230 and $490 \mathrm{kHz}$ (F. Wyrowski, priv. comm.). In P102, the shift for the FLASH345 observations was solved, however the FLASH460 observations still had the same shift of $490 \mathrm{kHz}$ (F. Wyrowski, priv. comm.). A correction was performed for these frequency shifts. The OFF position used in P101 and P102 is at $\alpha_{(2000)}=12^{\mathrm{h}} 25^{\mathrm{m}} 15^{\mathrm{s}}$ and $\delta_{(2000)}=-71^{\circ} 15^{\prime} 21^{\prime \prime}$, which is free of ${ }^{12} \mathrm{CO}(3-2)$ emission with a baseline rms of $T_{\mathrm{mb}}=0.04 \mathrm{~K}$ within $0.06 \mathrm{~km} \mathrm{~s}^{-1}$.

The observations with FLASH345 have a spectral resolution of $0.033 \mathrm{~km} \mathrm{~s}^{-1}$ and an angular resolution of $\sim 18^{\prime \prime}$. The FLASH460 observations have a spectral resolution of $0.05 \mathrm{~km} \mathrm{~s}^{-1}$ and an angular resolution of $\sim 14^{\prime \prime}$. For further analysis, all the observations (both with PI230 and $\mathrm{FLASH}^{+}$) were sampled at the same spectral resolution of $0.1 \mathrm{~km} \mathrm{~s}^{-1}$ since this is sufficient for Musca while it reduces the rms of $\mathrm{CO}(4-3)$ to $\sim 0.2 \mathrm{~K}$ and $\sim 0.5 \mathrm{~K}$ for the northern and southern map, respectively. This difference in data quality is due to different weather conditions with the water vapour varying between $\mathrm{pwv}=0.4$ and $\mathrm{pwv}=1.0$. Generally speaking, the observations for the northern map were carried out under better weather conditions. The main beam efficiencies ${ }^{3}$ used for the FLASH345 and FLASH460 observations are $\eta_{\mathrm{mb}}=0.65$ and $\eta_{\mathrm{mb}}=0.49$, respectively. The results from the ${ }^{12} \mathrm{CO}(3-2)$ and ${ }^{12} \mathrm{CO}(4-3)$ transitions are presented in the companion paper (Bonne et al. 2020, hereafter Paper II), while the ${ }^{13} \mathrm{CO}(3-2)$ data will be discussed in this article.

\subsection{NANTEN2: ${ }^{12} \mathrm{CO}(1-0)$ in Musca}

To obtain a more general view of the Musca cloud, we use ${ }^{12} \mathrm{CO}(1-0)$ observations of the Musca cloud with the NANTEN2 telescope at Pampa la Bola in the Atacama desert. These observations were carried out with the single-beam SIS receiver on the telescope, developed by Nagoya University, with a $1 \mathrm{GHz}$ bandwidth and a spectral resolution of $0.16 \mathrm{~km} \mathrm{~s}^{-1}$ at $115 \mathrm{GHz}$. The observations made several 30' OTF maps, resulting in a full map size of 9 square degrees $(7.3 \mathrm{pc} \times 7.3 \mathrm{pc}$ at a distance of $140 \mathrm{pc})$ with an rms noise of $0.45 \mathrm{~K}$ within the spectral resolution of $0.16 \mathrm{~km} \mathrm{~s}^{-1}$. This large map covers the Musca filament as well as the extended ambient cloud. The region of the Musca cloud covered by the NANTEN2 observations is indicated in Fig. 2. The main beam temperature scale was calibrated with Orion KL to be $52.6 \mathrm{~K}$, such that the observations are consistent with the intensity of the CfA $1.2 \mathrm{~m}$ telescope. The data was reduced with a linear baseline fit to the emission-free part of the spectrum.

\footnotetext{
http://www . apex-telescope.org/telescope/efficiency/
} 

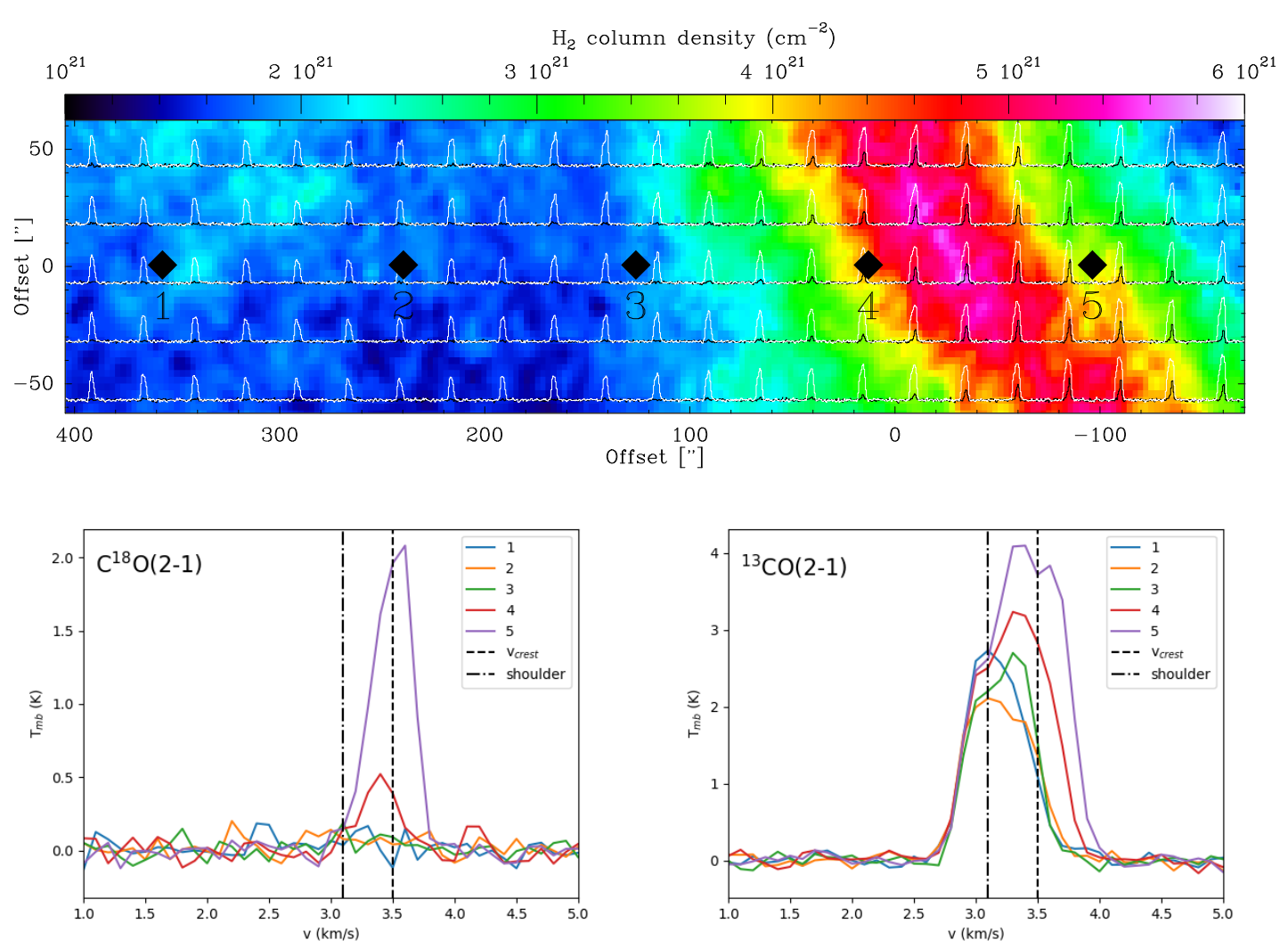

Fig. 6. Top: ${ }^{13} \mathrm{CO}(2-1)$ (in white) and $\mathrm{C}^{18} \mathrm{O}(2-1)$ (in black) spectra overlayed on the Herschel column density map of Musca (Cox et al. 2016) for the northern position. The offset is centred on: $\alpha_{(2000)}=12^{\mathrm{h}} 28^{\mathrm{m}} 58^{\mathrm{s}}$ and $\delta_{(2000)}=-71^{\circ} 16^{\prime} 55^{\prime \prime}$, and $100^{\prime \prime}$ corresponds to $\sim 0.07 \mathrm{pc}$ at a distance of $140 \mathrm{pc} . \mathrm{C}^{18} \mathrm{O}$ emission is only detected at the crest (green-yellow-red), while ${ }^{13} \mathrm{CO}$ can be used to trace the strands (blue) as well. Bottom right: several ${ }^{13} \mathrm{CO}(2-1)$ spectra are displayed that were extracted at the indicated positions in the map above. This shows that ${ }^{13} \mathrm{CO}$ has two components towards the filament crest. Bottom left: same for $\mathrm{C}^{18} \mathrm{O}(2-1)$, demonstrating it is only detected towards the filament crest.

\subsection{Herschel and extinction maps}

The column density and dust temperature profile close to the filament are provided by data from the Herschel Gould Belt Survey (HGBS) ${ }^{4}$ (André et al. 2010; Cox et al. 2016). For a more extended view on the mass distribution in the Musca cloud, we derived an extinction map of the region using the code $A_{\mathrm{v}} M a p$ (Schneider et al. 2011) which measures the average reddening of background stars in the 2MASS catalogue. The map was smoothed to a resolution of $5^{\prime}$ in order to improve the quality of the extinction map. In Fig. 2 the high resolution Herschel column density map (18.2") is embedded in the extinction map. In order to combine the Herschel and large scale extinction data, a linear relation between the Herschel column density and extinction was fitted. A conversion $N_{\mathrm{H}_{2}}=0.83 \pm 0.02 \times 10^{21} A_{\mathrm{v}}$ gave the best fit, which allowed to convert extinction to column density based on the information in the Herschel map. This conversion factor from extinction to column density for Musca is close to the canonical values reported for large samples in the galaxy (e.g. Bohlin et al. 1978; Güver \& Özel 2009; Rachford et al. 2009).

\section{Results}

In this section, the first results from the $\mathrm{CO}(2-1)$ isotopologues are presented. The emission from these isotopologues in the northern and southern APEX map is presented in Figs. 6 and 7, while the average spectra towards the filament crest are presented in Fig. 8. The observations will be presented step by step, starting

\footnotetext{
4 http://gouldbelt-herschel. cea.fr/archives
}

with $\mathrm{C}^{18} \mathrm{O}$, followed by ${ }^{13} \mathrm{CO}$ and in the end ${ }^{12} \mathrm{CO}$, because it can be observed in the before mentioned figures that the spectra show increasing complexity from $\mathrm{C}^{18} \mathrm{O}$ to ${ }^{12} \mathrm{CO}$.

\section{1. $C^{18} \mathrm{O}$ emission from the filament crest}

Figures 6 and 7 show the ${ }^{13} \mathrm{CO}(2-1)$ and $\mathrm{C}^{18} \mathrm{O}(2-1)$ spectra of the northern and southern map overlaid on the Herschel column density. In both maps, $\mathrm{C}^{18} \mathrm{O}(2-1)$ is only detected at the crest of the Musca filament within a physical size of $0.1-0.15 \mathrm{pc}$, and the brightness of $\mathrm{C}^{18} \mathrm{O}(2-1)$ quickly decreases when moving towards the strands around the filament crest. It suggests that $\mathrm{C}^{18} \mathrm{O}$ is tracing only the highest column density regions of the filament (typically $N_{\mathrm{H}_{2}}>3 \times 10^{21} \mathrm{~cm}^{-3}$ ).

Inspecting the spectra in both maps, we confirm that the filament crest has a single velocity component with a transonic linewidth of $0.15 \mathrm{~km} \mathrm{~s}^{-1}$, going up to $0.2 \mathrm{~km} \mathrm{~s}^{-1}$, as reported in Hacar et al. (2016). However, it should be noted that Fig. 8 shows that the $\mathrm{C}^{18} \mathrm{O}$ emission in the southern map has a pronounced shoulder which might be the result of a second velocity component. Additionally, we note a slight and continuous shift of the central velocity across the crest. To further investigate this velocity structure, a gaussian line profile was fitted to the $\mathrm{C}^{18} \mathrm{O}(2-1)$ spectra above the $3 \sigma$ noise rms. The velocity field obtained from the fitting is presented in Figs. 9 and 10. These figures demonstrate organised velocity fields across the crest in both maps with a typical velocity interval of $\sim 0.2 \mathrm{~km} \mathrm{~s}^{-1}$, which is similar to the spectral linewidth, suggesting a part of the linewidth might be due to these gradients. The velocity gradients were calculated 

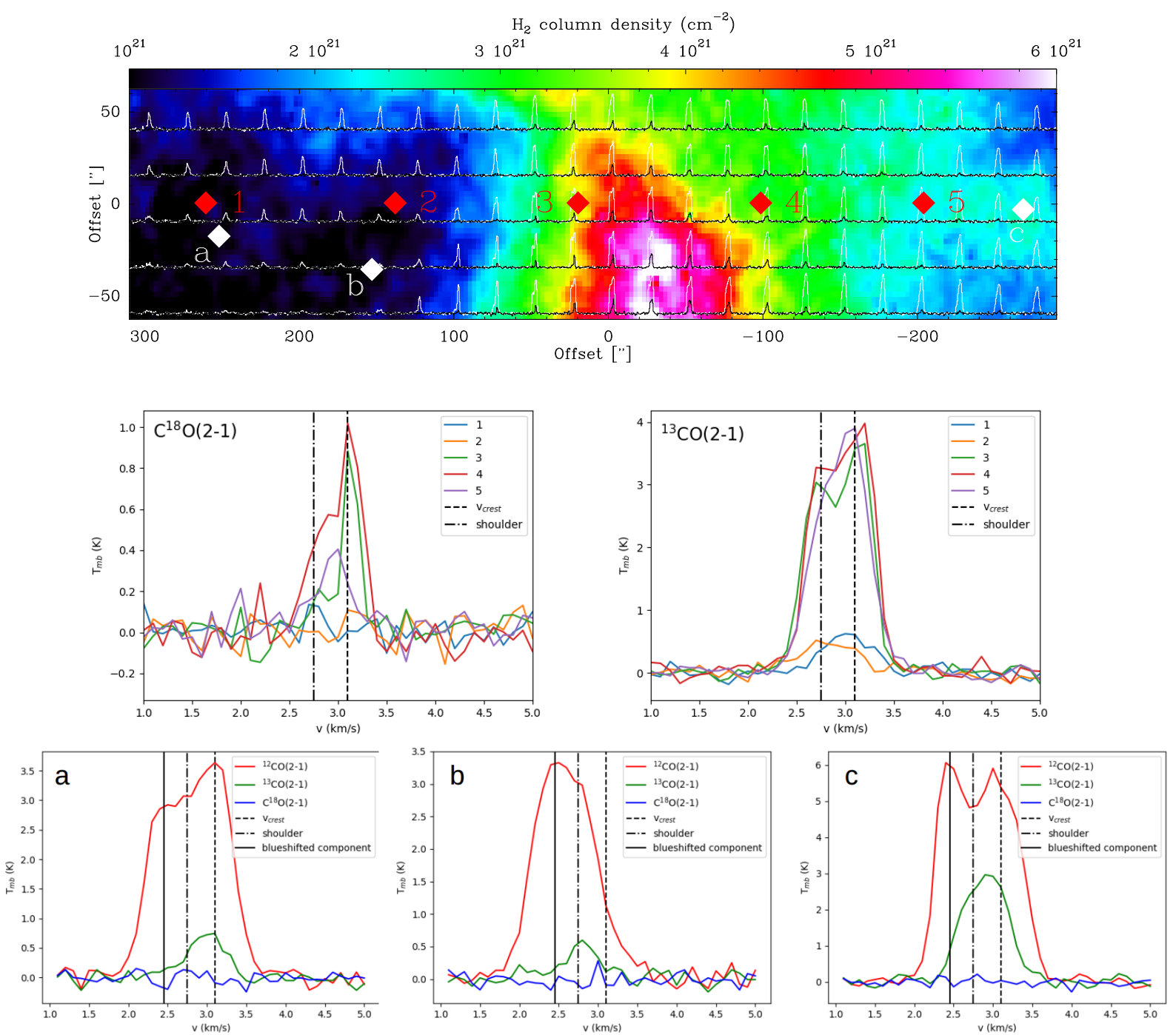

Fig. 7. Top and middle: same as Fig. 6, but for the southern position, where the offset is centered on: $\alpha_{(2000)}=12^{\mathrm{h}} 24^{\mathrm{m}} 46^{\mathrm{s}}$ and $\delta_{(2000)}=$ $-71^{\circ} 47^{\prime} 20^{\prime \prime}$. It can be noted that at the location where there are no strands $\left(N_{\mathrm{H}_{2}}<1.5 \times 10^{21} \mathrm{~cm}^{-2}\right)$ that the ${ }^{13} \mathrm{CO}$ emission also disappears. Bottom: ${ }^{12} \mathrm{CO}(2-1),{ }^{13} \mathrm{CO}(2-1)$ and $\mathrm{C}^{18} \mathrm{O}(2-1)$ emission at the indicated white positions $(\mathrm{a}, \mathrm{b}, \mathrm{c})$ in the map above with the same beam size $\left(28^{\prime \prime}\right)$ and spectral resolution $\left(0.1 \mathrm{~km} \mathrm{~s}^{-1}\right)$. This indicates that in ${ }^{12} \mathrm{CO}(2-1)$ there is an extra blueshifted component that is barely detected in ${ }^{13} \mathrm{CO}(2-1)$. It also shows that $\mathrm{C}^{18} \mathrm{O}(2-1)$ is not detected away from the filament crest with this data.

using the nearest neighbour values for every pixel. They have a magnitude of 1.6 and $2.4 \mathrm{~km} \mathrm{~s}^{-1} \mathrm{pc}^{-1}$ and an angle of $77^{\circ}$ and $45^{\circ}$ compared to the local orientation of the filament crest for the northern and southern map, respectively. One should note that the southern map covers a part of the filament that is fragmenting which might have an impact on the observed velocity gradient (e.g Hacar \& Tafalla 2011; Williams et al. 2018; Arzoumanian et al. 2018). We also find that the central velocity at the crest is $\sim 3.5 \mathrm{~km} \mathrm{~s}^{-1}$ in the north and around $3.0-3.1 \mathrm{~km} \mathrm{~s}^{-1}$ in the south.

Since the velocity gradient is in the opposite direction in the south compared to the north, see Fig. 10, this does not lead to a straightforward analysis of the entire Musca filament crest as a simple rotating filament which is theoretically studied in Recchi et al. (2014).

\section{2. ${ }^{13} \mathrm{CO}$ emission from the strands}

In contrast to $\mathrm{C}^{18} \mathrm{O}(2-1)$, the emission of ${ }^{13} \mathrm{CO}(2-1)$ is not confined to the filament crest alone, but is also high towards the strands of Musca, see Figs. 6, 7 and 11. At the locations where the strands are not bright in the Herschel dust column density map, the ${ }^{13} \mathrm{CO}$ emission also strongly decreases, see Figs. 7 and 11.

Inspecting the ${ }^{13} \mathrm{CO}(2-1)$ spectra of both maps in more detail, a component at the same velocity as the filament crest can be found, as well as a slightly blueshifted shoulder at $\mathrm{v}_{\text {los }} \sim 3.1$ $\mathrm{km} \mathrm{s}^{-1}$ in the northern map and $\mathrm{v}_{\text {los }} \sim 2.7-2.8 \mathrm{~km} \mathrm{~s}^{-1}$ in the southern map, see Fig. 8. The velocity component related to the filament crest disappears when moving away from the filament crest, while the emission from the shoulder remains present over the strands, see e.g. Figs. 6 and 11. This implies that the observed shoulder comes from slightly more blueshifted emission related to the strands. From this moment on, when talking about the shoulder we thus implicitly talk about the strands and vice versa.

The velocity field of $\mathrm{C}^{18} \mathrm{O}(2-1)$ demonstrated internal motion in the filament crest. The ${ }^{13} \mathrm{CO}(2-1)$ spectra provide more information, namely that there are two velocity components: one related to the filament crest and a second component related to the strands.

At this point, we have presented the blueshifted shoulder that correlates well with the Herschel strands in both maps. 

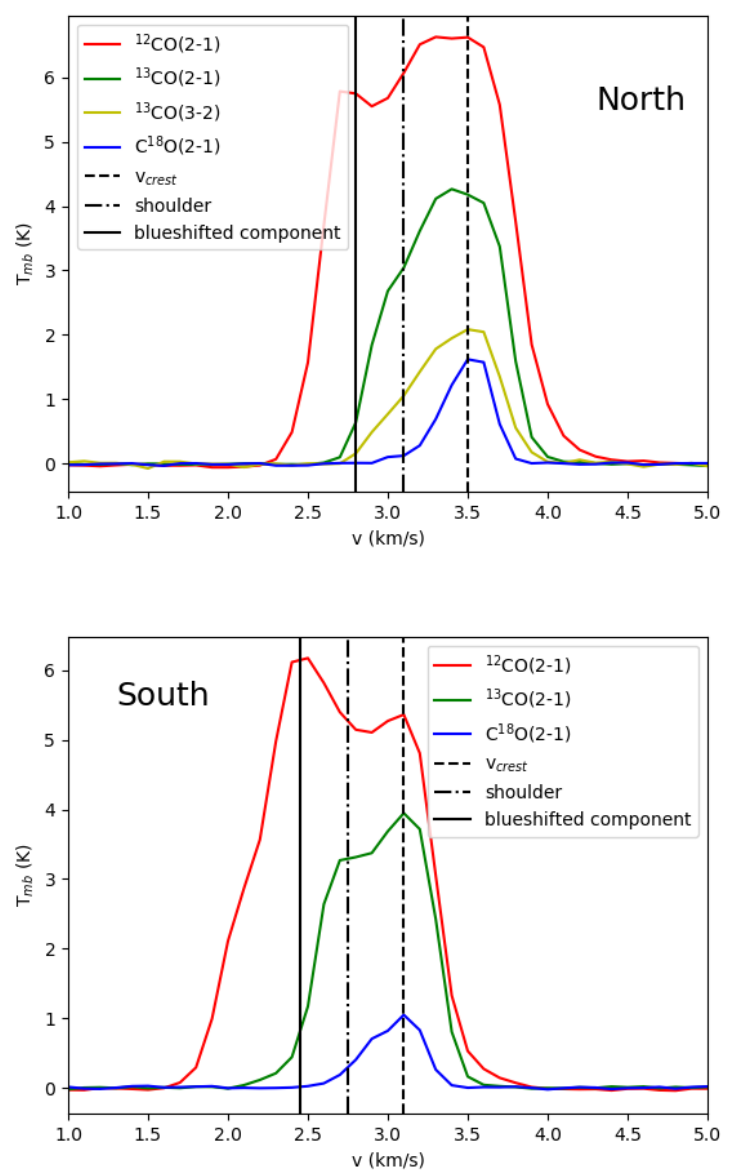

Fig. 8. Top: averaged $\mathrm{CO}(2-1)$ isotopologue spectra and the ${ }^{13} \mathrm{CO}(3-$ 2) spectrum observed towards the filament crest $\left(N_{\mathrm{H}_{2}}>3 \times 10^{21} \mathrm{~cm}^{-2}\right)$ in the northern map, showing the presence of the three components. Bottom: same for the crest of the southern map.

This indicates that the strands around the filament crest are blueshifted compared to the filament crest. When inspecting the ${ }^{13} \mathrm{CO}(2-1)$ channel maps of the southern map in Fig. 11 in more detail, one finds that east of the filament crest there is some weak ${ }^{13} \mathrm{CO}(2-1)$ emission $\left(T_{\mathrm{mb}} \lesssim 1 \mathrm{~K}\right)$ away from the filament crest at more or less the same velocity as the filament crest, see Figs. 7 and 11. This emission suggests that at some locations there is also a small amount of "redshifted" gas near the filament crest that is roughly at the same velocity along the line of sight as the filament crest itself.

\section{3. ${ }^{12}$ CO: Blueshifted emission}

Inspecting the APEX ${ }^{12} \mathrm{CO}(2-1)$ spectra, another velocity component shows up with little corresponding emission in ${ }^{13} \mathrm{CO}(2-1)$, see e.g. Figs. 7 and 8. This new velocity component, which is even more blueshifted than the shoulder, is observed both in the northern and southern map and also at the locations where the strands disappear (in the Herschel column density map), see Fig. 7. This suggests, as ${ }^{13} \mathrm{CO}(2-1)$ is only marginally detected at this velocity, that there is low column density gas present around the Musca filament with blueshifted velocities along the line of sight of $2.7 \mathrm{~km} \mathrm{~s}^{-1}$ in the north and $2.5 \mathrm{~km} \mathrm{~s}^{-1}$ in the south, see Fig. 8. We refer to it as "the blueshifted component" from now on.

These observations of the CO isotopologues thus indicate that the Musca cloud has two more velocity components on

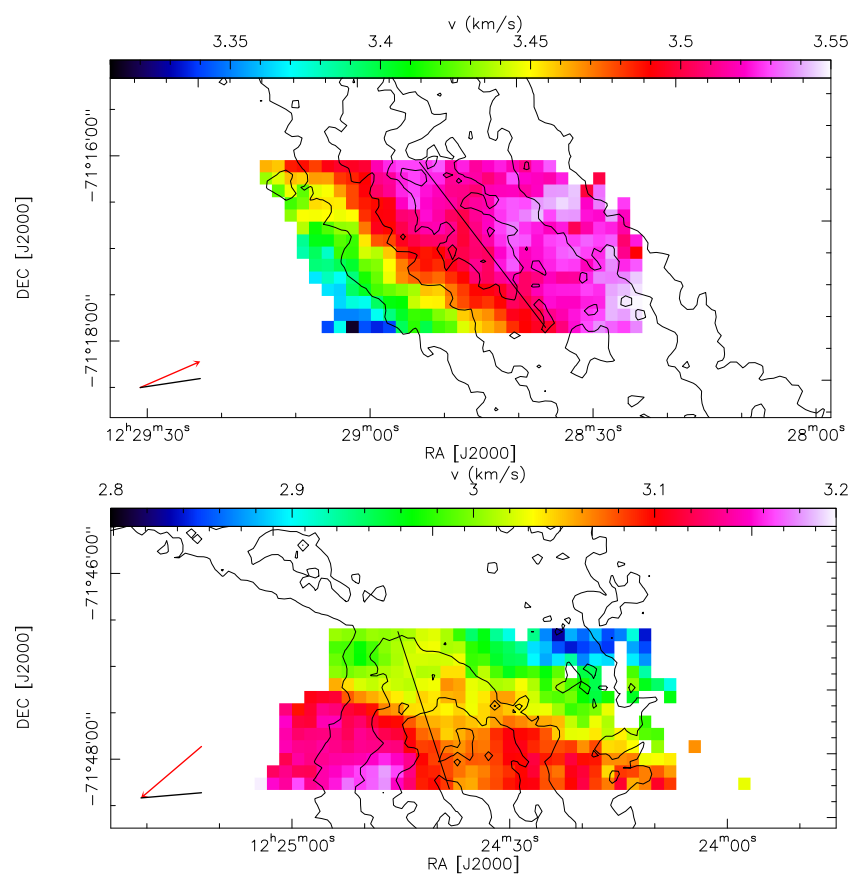

Fig. 9. Velocity field obtained over the Musca filament crest from the $\mathrm{C}^{18} \mathrm{O}(2-1)$ line in the northern (top) and southern (bottom) map. The contours indicate the column density levels of the filament crest $\left(N_{\mathrm{H}_{2}}=\right.$ $\left.3-6 \times 10^{21} \mathrm{~cm}^{-3}\right)$. The black line on the velocity field connects the maximal column density at the top and bottom of the map, indicating the local orientation of the filament axis. In the lower left corner, the red arrow indicates the orientation of the velocity gradient, while the black line indicates the orientation of the magnetic field.

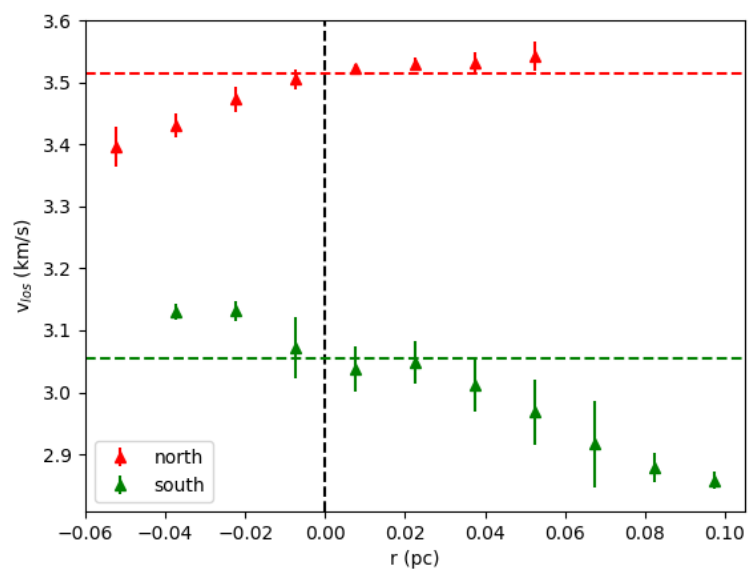

Fig. 10. Line-of sight velocity across the filament derived from $\mathrm{C}^{18} \mathrm{O}(2-$ 1) emission averaged along the filament as a function of the distance ( $r$ ) for the northern (red) and southern (green) maps. The distance is always determined perpendicular to the local orientation of the filament crest center. The horizontal dashed lines indicate the velocity at the center of the filament crest, the vertical dashed line indicates the center of the crest, and the errorbars indicate the dispersion at each radius in the maps.

top of the already established velocity-coherent filament crest: the shoulder (detected in ${ }^{13} \mathrm{CO}$ ) and the blueshifted velocity component (detected in ${ }^{12} \mathrm{CO}$ ). These different velocities can also be seen as a continuous velocity structure from large scale blueshifted gas to the small scale crest which corresponds to the reddest $\mathrm{CO}$ emission of the region. This generally indicates that the kinematics in the Musca cloud is more complex than 

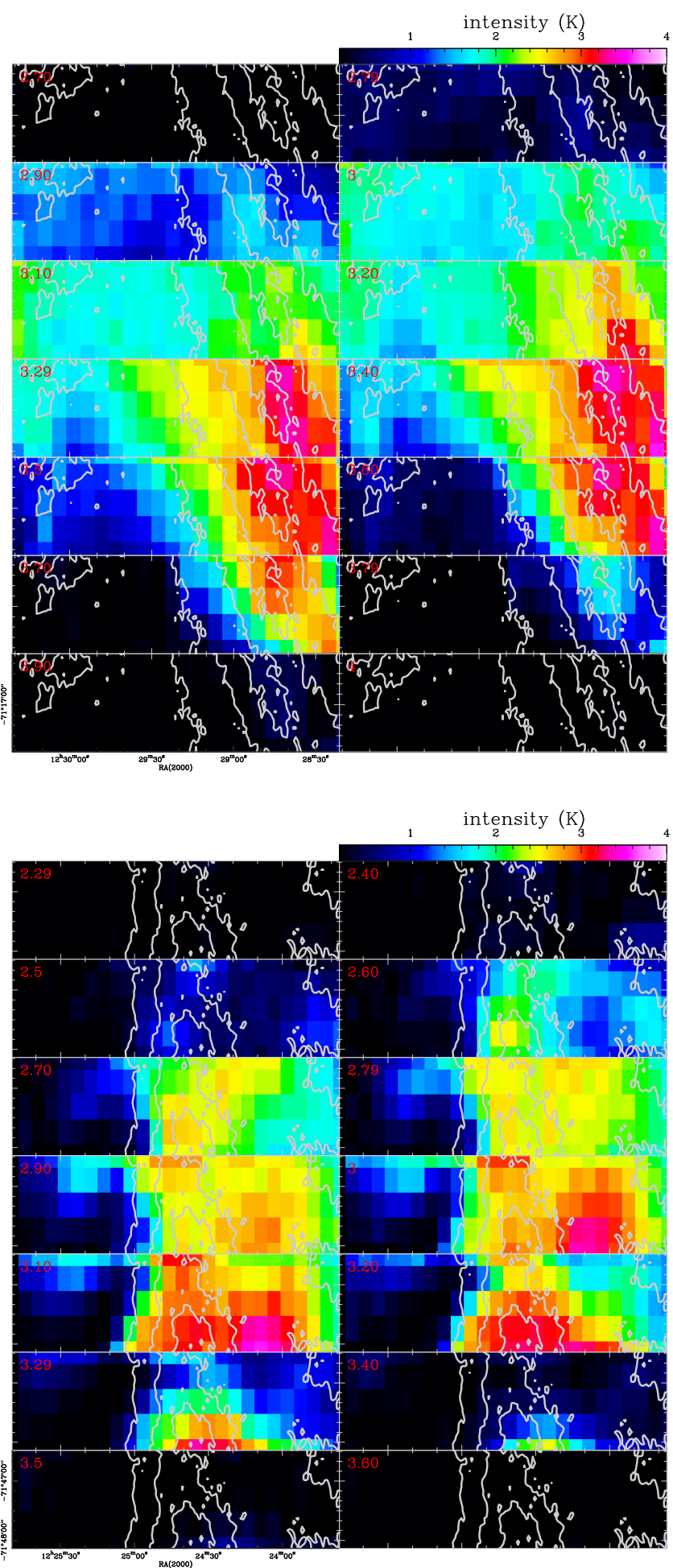

Fig. 11. Top: channel maps of the APEX ${ }^{13} \mathrm{CO}(2-1)$ observations in the northern map. The contours indicate the Herschel column densities $N_{\mathrm{H}_{2}}=2 \times 10^{21}, 3.5 \times 10^{21}$ and $5 \times 10^{21} \mathrm{~cm}^{-2}$. The velocity of the channel is indicated in red at the upper left corner of every channel. It can be seen that the velocities of the blueshifted shoulder trace the strands. Bottom: same for the southern map.

the single velocity component of the filament crest (Kainulainen et al. 2016; Hacar et al. 2016), and that large scale kinematics might play an important role in the filament formation and cloud evolution.

\subsection{NANTEN2 ${ }^{12} \mathrm{CO}(1-0)$ data}

The ${ }^{12} \mathrm{CO}(1-0)$ mapping performed by the NANTEN2 telescope provides a view on the large scale kinematics of the Musca filament and the ambient cloud. Figure 12 presents channel maps of this data set, which shows that the brightest ${ }^{12} \mathrm{CO}(1-0)$ gas is located at the crest and strands defined by the Herschel data.

In the channel maps with $v<2.7 \mathrm{~km} \mathrm{~s}^{-1}$, there is also emission observed outside the filament contours. This is confirmed with the velocity field obtained from the NANTEN2 data in Fig. 13, which demonstrates that the blueshifted component traces a more diffuse and extended ambient cloud that is observed in dust continuum emission with Herschel and in the extinction map. At velocities between 2.7 and $4 \mathrm{~km} \mathrm{~s}^{-1}$, corresponding to the velocity of the shoulder and crest, the emission is nicely constrained to the filament seen by Herschel as was already inferred from the APEX data, see Fig. 12.

The NANTEN2 observations confirm that the large scale, surrounding gas of the Musca filament is mostly blueshifted. It also confirms that smaller scale blueshifted strands can be found at both sides of the filament crest, as was already suggested from the APEX data. This indicates that there are local changes in the position of these blueshifted strands compared to the crest. The column density profile perpendicular to the filament crest in Fig. 14 also shows that the filament crest, both in the south and north, is not located at the center of the strands. It even shows that the crest has a direct border with the more diffuse ambient cloud at some locations, which results in locally asymmetric column density profiles.

Furthermore, NANTEN2 data shows that there is virtually no $\mathrm{CO}$ emission in the whole Musca region that is redshifted compared to the crest at $3.0-3.5 \mathrm{~km} \mathrm{~s}^{-1}$. This confirms the tendency already noted at small scales with the APEX data in the two maps. In conclusion, the Musca filament and its possible gas reservoir have an interesting asymmetric distribution both spatially (crest concentrated in the west/north-west direction) and kinematically (redshifted crest and strands compared to the blueshifted large-scale ambient cloud).

\subsection{Orientation of the velocity gradient and the magnetic field}

An organised velocity gradient over the Musca filament crest was reported in Sect. 4.1 from $\mathrm{C}^{18} \mathrm{O}$ observations. Here, the orientation of this velocity gradient is compared to the orientation of the local magnetic field. For the entire Musca filament, the magnetic field is nearly perpendicular to the filament crest (Pereyra \& Magalhães 2004; Planck Collaboration Int. XIII 2016), with a typical angle between the filament crest and the magnetic field of $80^{\circ}$ (Cox et al. 2016).

In order to further investigate a possible link between the plane of the sky magnetic field orientation and the velocity gradient in the filament crest, the magnetic field was constructed from the Planck $353 \mathrm{GHz}$ data (Planck Collaboration I 2016). To reduce the noise in the magnetic field map, the I, Q and $\mathrm{U}$ maps were smoothed to a resolution of $10^{\prime}$ (or $0.4 \mathrm{pc}$ for Musca at 140 pc) (Planck Collaboration Int. XXXV 2016; Planck Collaboration Int. XIII 2016). The orientation of the magnetic field was then calculated in the IAU convention using the formulas presented in Planck Collaboration Int. XXXV (2016) and Planck Collaboration Int. XIII (2016). In the area covered by the $\mathrm{C}^{18} \mathrm{O}$ velocity maps, the magnetic field orientation (Planck Collaboration Int. XIII 2016) and the average velocity gradient were compared in Fig. 9, showing no clear correlation. In the northern map, the angle between the magnetic field orientation 


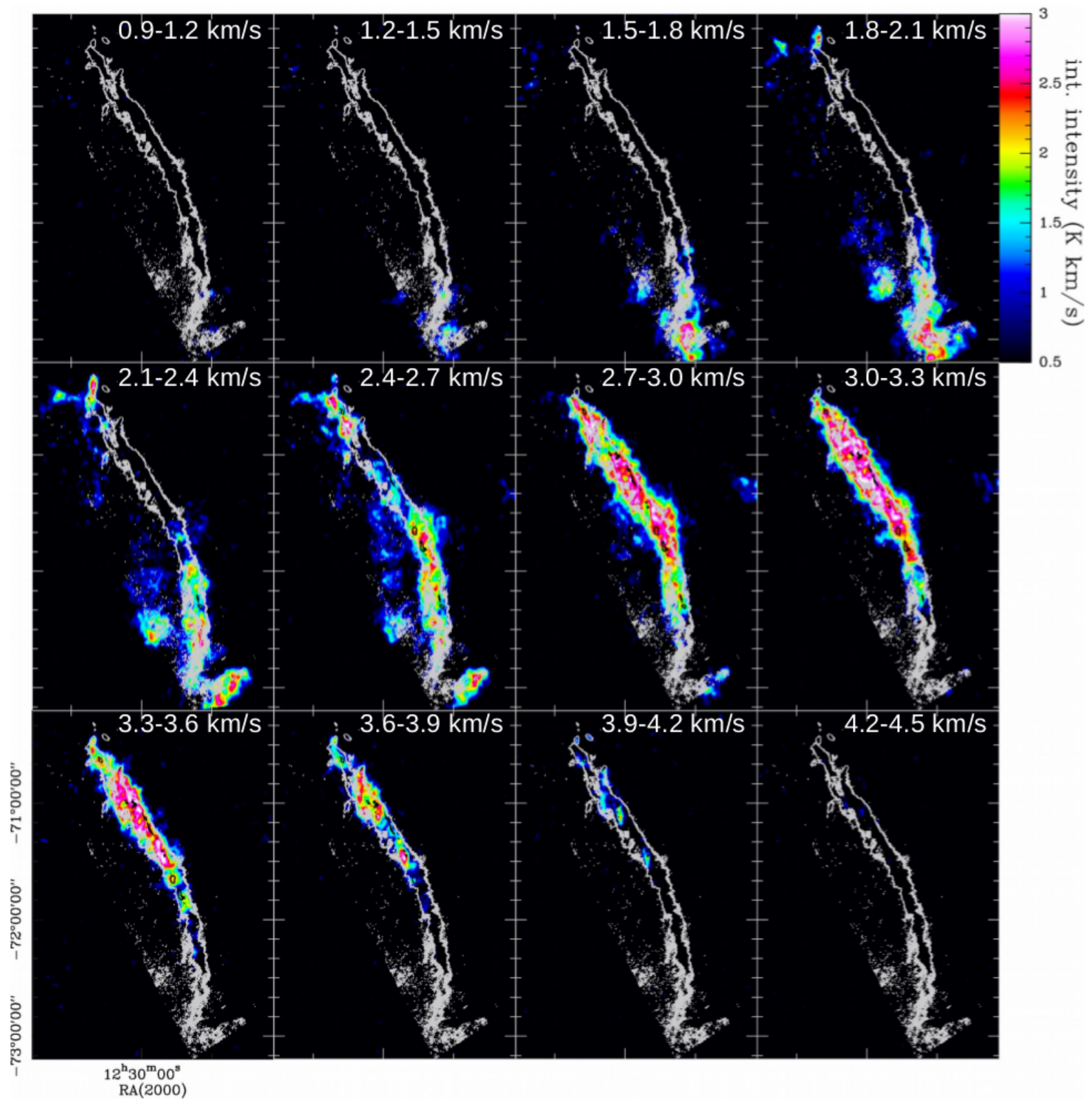

Fig. 12. Integrated intensity of NANTEN2 data over velocity intervals of $0.3 \mathrm{~km} \mathrm{~s}^{-1}$. The black contours at $N_{\mathrm{H}_{2}}=5 \times 10^{21} \mathrm{~cm}^{-2}$ from the Herschel data indicate the center of the filemant crest. These black contours are best visible at velocities between 2.7 and $3.6 \mathrm{~km} \mathrm{~s}^{-1}$ when there is ${ }^{12} \mathrm{CO}(1-0)$ emission towards the crest. The grey contour, at $N_{\mathrm{H}_{2}}=2 \times 10^{21} \mathrm{~cm}^{-2}$, indicates the area that encloses the strands. It can be observed that there is some extended emission east of the Musca filament and that the filament crest is not at the middle of the area containing the strands.

and the average velocity gradient is $28^{\circ}$. In the southern map the angle is $54^{\circ}$. A word of caution on this comparison: the velocity gradient is located over the filament crest, while the resolution of the Planck magnetic field orientation is significantly larger than the $\sim 0.1 \mathrm{pc}$ size of the filament crest. It does however show that the velocity gradient over the filament crest has a significant offset compared to the large scale organised magnetic field in its close surroundings.

\section{Line radiative transfer analysis}

\subsection{Density in Musca from radiative transfer of $\mathrm{CO}$}

With the non-LTE line radiative transfer code RADEX (van der Tak et al. 2007), which uses the LAMBDA database (Schöier et al. 2005), we investigate whether the CO isotopologue data can provide a consistent picture for the 3D geometry of the Musca filament. To do this, we begin with estimating the density profile across the Musca filament. In Fig. 15 it is shown that we can estimate the density with RADEX using the ${ }^{13} \mathrm{CO}(3-2) /{ }^{13} \mathrm{CO}(2-1)$ brightness ratio, after smoothing ${ }^{13} \mathrm{CO}(3-2)$ to the same resolution of ${ }^{13} \mathrm{CO}(2-1)$ (here $\left.28^{\prime \prime}\right)$, because this ratio strongly depends on the density. In particular between $10^{2}$ and $5 \times 10^{4} \mathrm{~cm}^{-3}$, which covers the wide range of proposed densities at the Musca crest (Kainulainen et al. 2016; Tritsis \& Tassis 2018), the ratio strongly depends on the density. We focus on the northern map since we only have ${ }^{13} \mathrm{CO}(3-2)$ and ${ }^{13} \mathrm{CO}(2-1)$ data for this location, which has a Herschel column density up to $N_{\mathrm{H}_{2}} \sim 6 \times 10^{21} \mathrm{~cm}^{-2}$, see Fig. 14.

In principle the line ratio does not depend on the abundance of ${ }^{13} \mathrm{CO}$, but since both ${ }^{13} \mathrm{CO}$ lines become optically thick at the crest this is not completely true. However, in Appendix B it is 

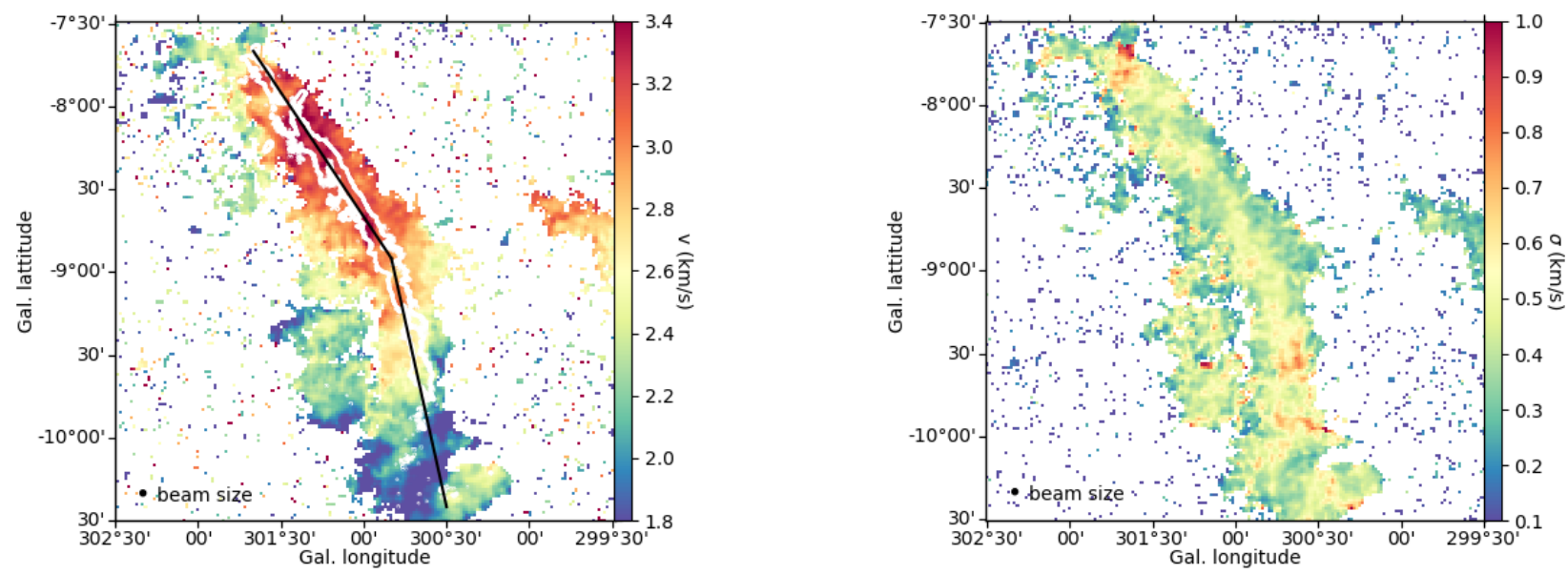

Fig. 13. Left: ${ }^{12} \mathrm{CO}(1-0)$ velocity field in the Musca cloud from fitting a single gaussian to the ${ }^{12} \mathrm{CO}(1-0)$ NANTEN2 data, showing an organised velocity field along the Musca filament. The black line indicates the central axis used to construct the PV diagram in Fig. 23 perpendicular to the Musca filament. The white contours show the high column density region $\left(N_{\mathrm{H}_{2}}>2 \times 10^{21} \mathrm{~cm}^{-2}\right)$ in Musca. Right: ${ }^{12} \mathrm{CO}(1-0)$ linewidth from the Gaussian fitting to the NANTEN2 data.
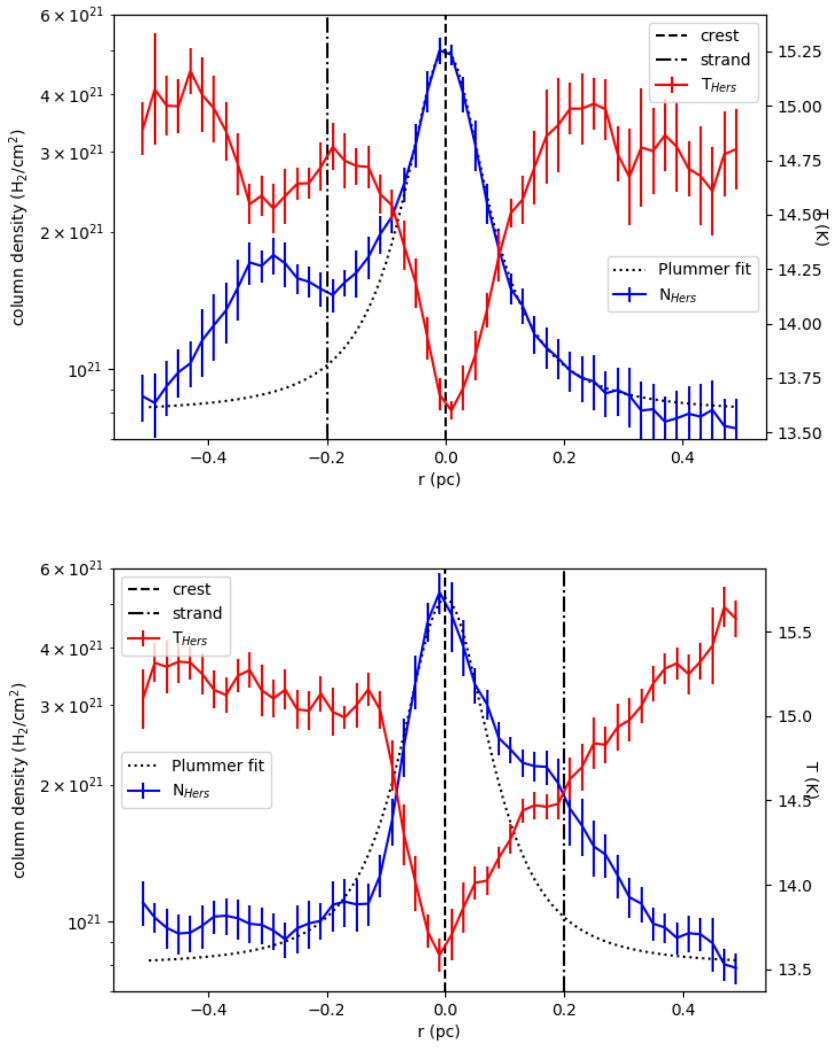

Fig. 14. Top: Herschel column density and temperature profile in the northern map. It demonstrates an asymmetry of the column density due to the strands. The fitted Plummer profile (excluding the strands) shows that the strand is a dense structure directly next to the filament crest. The standard deviation from the average value is also indicated at each radius. A negative radius was chosen to be located east of the filament crest. Bottom: same for the southern map, note that the strand has changed side.

demonstrated that the varying optical depth related to the column density or abundance variations is not the main uncertainty for the ${ }^{13} \mathrm{CO}(3-2) /{ }^{13} \mathrm{CO}(2-1)$ ratio.

To construct the density profile, a RADEX grid of the ${ }^{13} \mathrm{CO}$ brightness temperature ratio was created with 40 points on a

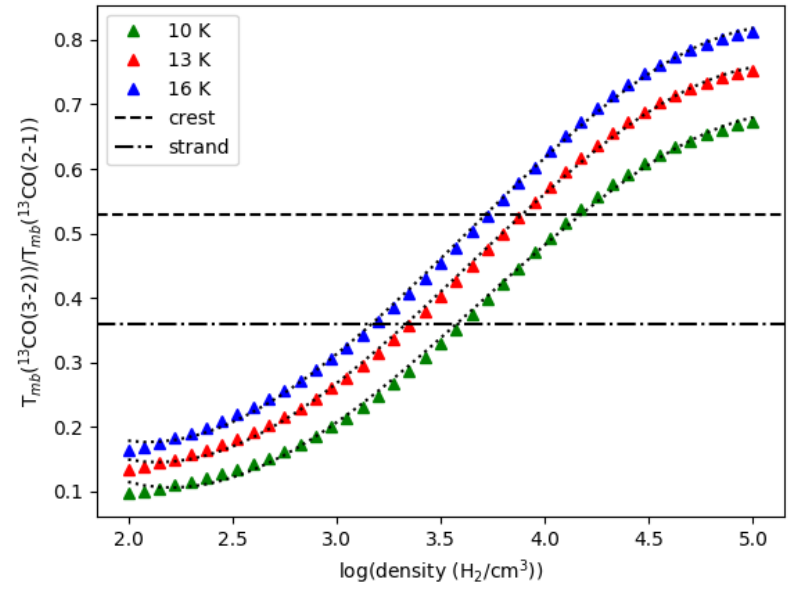

Fig. 15. Evolution of the ${ }^{13} \mathrm{CO}(3-2) /{ }^{13} \mathrm{CO}(2-1)$ brightness temperature ratio as a function of density for realistic temperatures of the Musca filament. The dotted curves are the functions fitted through the RADEX results to invert the observed ratio in Musca to a density. The horizontal lines indicate the average observed ratios for the strand and the crest in the northern map.

$\log$ scale between $n_{\mathrm{H}_{2}}=10^{2}$ and $10^{5} \mathrm{~cm}^{-3}$ for three different kinetic temperatures: 10,13 , and $16 \mathrm{~K}$. This covers the range of Herschel dust temperatures as well as kinetic gas temperatures put forward for Musca by Machaieie et al. (2017). The full width at half maximum (FWHM) and column density for ${ }^{13} \mathrm{CO}$ that are used in the RADEX calculations are $0.7 \mathrm{~km} \mathrm{~s}^{-1}$ and $N_{13} \mathrm{CO}=1.1 \times 10^{16} \mathrm{~cm}^{-2}$, respectively, which is obtained from $N_{\mathrm{H}_{2}}=6 \times 10^{21} \mathrm{~cm}^{-2}$ using $\left[\mathrm{H}_{2}\right] /\left[{ }^{13} \mathrm{CO}\right]=5.7 \times 10^{5}$ (from $\left[\mathrm{H}_{2}\right] /\left[{ }^{12} \mathrm{CO}\right] \sim 10^{4}$ and $\left[{ }^{12} \mathrm{CO}\right] /\left[{ }^{13} \mathrm{CO}\right] \sim 57$ Langer \& Penzias 1990). The calculated opacities by RADEX for these models vary between 3 and 6 for ${ }^{13} \mathrm{CO}(2-1)$ and 1 to 3 for ${ }^{13} \mathrm{CO}(3-2)$, which fits with the estimated opacities in Hacar et al. (2016).

To invert the observed ratios with APEX to a density profile, we work with the Herschel temperature profile in Fig. 14. The resulting density profile for the Musca filament is presented in Fig. 16.

This density profile predicts $n_{\mathrm{H}_{2}}=6.5 \pm 1.9 \times 10^{3} \mathrm{~cm}^{-3}$ at the filament crest that drops to $n_{\mathrm{H}_{2}} \sim 1-3 \times 10^{3} \mathrm{~cm}^{-3}$ for the strands, 


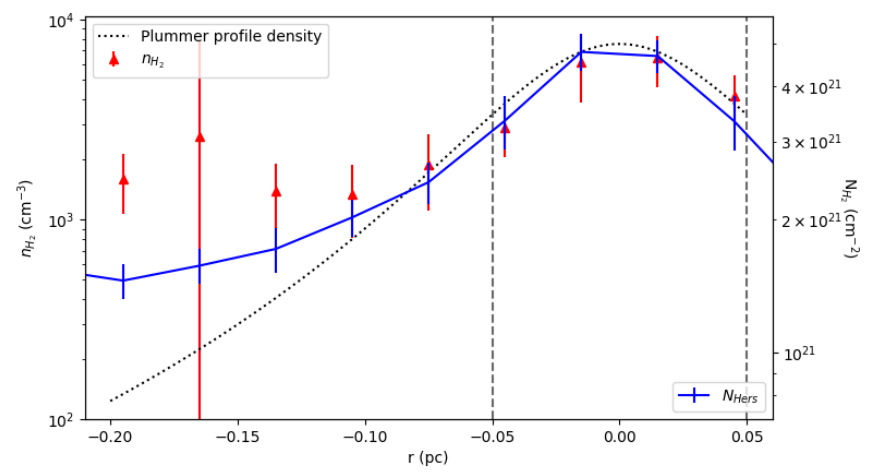

Fig. 16. Density profile and its standard deviation obtained from the ${ }^{13} \mathrm{CO}(3-2) /{ }^{13} \mathrm{CO}(2-1)$ ratio as a function of the distance from the filament crest for the northern map (in red). The vertical dashed lines indicate the extent of the filament crest. It should be noted that the Herschel temperature is too high for the filament crest such that the density will be slightly higher than the values shown in this figure, see Fig. 15. The dotted black line describes the Plummer density profile for the Musca filament as derived from the column density profile using Eq. (1) in Arzoumanian et al. (2011). This density profile fits with density predictions at the filament crest, but towards the strands the density is significantly higher than predicted from the Plummer profile. The Herschel column density profile, in blue, is plotted on the axis on the right. When comparing the density profile with the column density profile, one should take into account the different scales of the left and right axes.

see Fig. 16. However, for the interior of the filament crest the $13 \mathrm{~K}$ temperature is certainly overestimated. It was shown by Nielbock et al. (2012) and Roy et al. (2014) that the temperature in the dense interior of different clouds is at least $3 \mathrm{~K}$ lower than the Herschel dust temperature on the sky. Using a temperature of $10 \mathrm{~K}$, which is actually also suggested by the LTE study of ${ }^{13} \mathrm{CO}(1-0)$ and $\mathrm{C}^{18} \mathrm{O}(1-0)$ by Machaieie et al. (2017) for the filament crest, one gets a more probable typical density in the crest of $n_{\mathrm{H}_{2}}=1.3 \pm 0.4 \times 10^{4} \mathrm{~cm}^{-3}$.

Combining the obtained densities with the Herschel column densities, one can estimate the size of the dense gas along the line of sight as a function of the distance from the filament crest. This is shown in Fig. 17, demonstrating a typical size of $0.2-0.5 \mathrm{pc}$ for the strands and a size of $\sim 0.25 \mathrm{pc}$ at the filament crest for the overestimated temperature of $13 \mathrm{~K}$. For the most probable $10 \mathrm{~K}$ temperature of the crest, we get a size of $\sim 0.1 \mathrm{pc}$ for the crest. We thus get for both the filament crest and strands that their characteristic sizes along the lines of sight are roughly the same as their sizes in the plane of the sky, which implies a cylindrical geometry.

The density profile from the ${ }^{13} \mathrm{CO}(3-2) /{ }^{13} \mathrm{CO}(2-1)$ brightness ratio, is compared in Fig. 16 with the predicted density profile from fitting a Plummer profile to the filament crest in the northern APEX map (Cox et al. 2016). This shows that a Plummer profile provides an acceptable fit for the filament crest. In the strands we find that the density estimate from ${ }^{13} \mathrm{CO}(3-$ $2) /{ }^{13} \mathrm{CO}(2-1)$ is significantly higher than the density predicted by the Plummer profile, similar to what is observed for the column density in Fig. 14. This suggests that the strands consist of denser gas than the surroundings, and it reinforces our earlier proposal that the strands are additional components on the top of the density (presumably plummer-like) profile of the filament crest.

We also verified the overall consistency of the obtained density results for the filament crest and strands by studying the observed brightness of the CO isotopologues, see Table 1.

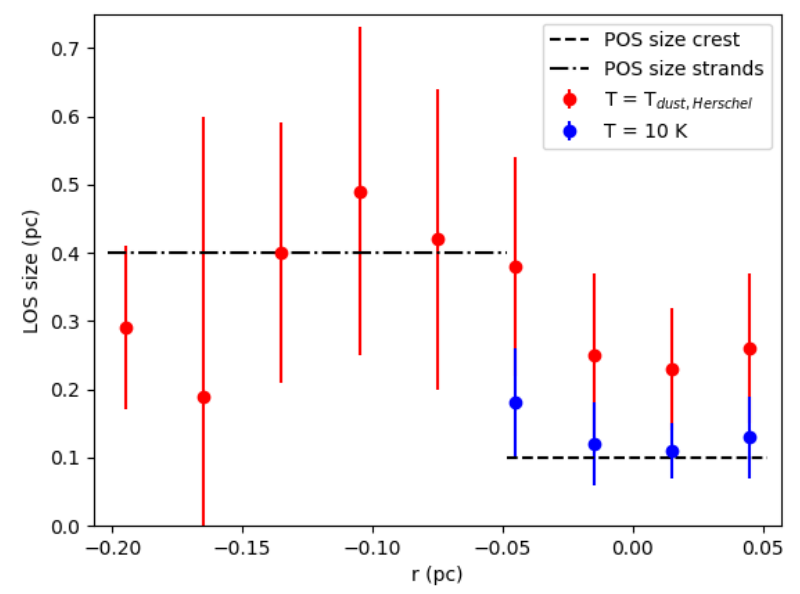

Fig. 17. Estimated characteristic size for the observed ${ }^{13} \mathrm{CO}$ emission in the line of sight as a function of the distance (r) from the center of the filament crest. The characteristic size is estimated combining the Herschel column density with the ${ }^{13} \mathrm{CO}(3-2) /{ }^{13} \mathrm{CO}(2-1)$ density estimate using the Herschel dust temperature (red) and a temperature of $10 \mathrm{~K}$ (blue). The horizontal lines indicate the characteristic sizes in the plane of the sky (POS) of the strands and the filament crest.

For the filament crest, we find that $n_{\mathrm{H}_{2}}=10^{4} \mathrm{~cm}^{-3}$ and $T_{\mathrm{k}}=$ $10 \mathrm{~K}$ reproduce the observed $\mathrm{CO}$ isotopologue brightnesses, even when taking into account a $30 \%$ uncertainty of the CO abundance as is shown in Table 1 . For the strands, we reproduce the observed ${ }^{13} \mathrm{CO}$ brightness with $n_{\mathrm{H}_{2}}=10^{3}-3 \times 10^{3} \mathrm{~cm}^{-3}$ and $T_{\mathrm{k}}=$ $15-18 \mathrm{~K}$, however it is not possible to reproduce the $\mathrm{C}^{18} \mathrm{O}$ brightness of the strands with typical $\left[{ }^{13} \mathrm{CO}\right] /\left[\mathrm{C}^{18} \mathrm{O}\right]$ ratios around 10. This will be addressed in more detail in the next section.

These results suggest that the kinetic temperature of the strands and the filament crest are similar to the Herschel dust temperature. Generally speaking it is found that $T_{\text {dust }} \leq T_{\mathrm{k}}$ (e.g. Goldsmith 2001; Merello et al. 2019), but in the dense interior of the Musca cloud $T_{\text {dust }} \approx T_{\mathrm{k}}$ can be expected (e.g. Goldsmith 2001; Galli et al. 2002; Ivlev et al. 2019). In the simulations by Seifried \& Walch (2016), the dust is not yet fully coupled to the gas at the predicted densities inside the filament crest $\left(n_{\mathrm{H}_{2}} \sim 10^{4} \mathrm{~cm}^{-3}\right)$, but in these simulations the dust temperature reaches values below $10 \mathrm{~K}$.

\section{2. $\mathrm{C}^{18} \mathrm{O}$ abundance drop in the strands}

Studying the $\mathrm{CO}$ isotopologue emission in the strands, a strong variation of the $\left[{ }^{13} \mathrm{CO}\right] /\left[\mathrm{C}^{18} \mathrm{O}\right]$ abundance ratio is observed. As already noted, the $\mathrm{C}^{18} \mathrm{O}(2-1)$ emission, with a typical rms of $\sim 0.07 \mathrm{~K}$, rapidly decreases in the strands until it is no longer clearly detected for a single beam in the strands. When studying the data in bins of $0.03 \mathrm{pc}$ as a function of the distance from the center of the filament crest, we always obtain a $>3 \sigma$ detection of $\mathrm{C}^{18} \mathrm{O}(2-1)$. This then allows to follow the rapid continuous increase of $\left[{ }^{13} \mathrm{CO}\right] /\left[\mathrm{C}^{18} \mathrm{O}\right]$ in Fig. 18 as a function of the distance from the filament crest, showing that the ratio varies by an order of magnitude over less than $0.2 \mathrm{pc}$. Figure 18 displays the evolution of the integrated line brightness ratio as a function of the distance, which is a decent proxy for the $\left[{ }^{13} \mathrm{CO}\right] /\left[\mathrm{C}^{18} \mathrm{O}\right]$ ratio because of the very similar excitation conditions for both lines. This strong increase of the $\left[{ }^{13} \mathrm{CO}\right] /\left[\mathrm{C}^{18} \mathrm{O}\right]$ ratio agrees with a weak $\mathrm{C}^{18} \mathrm{O}(2-1)$ detection at the velocity of the strands with a brightness of $\sim 0.08 \mathrm{~K}$ when smoothing over the area covered by the strands, while the brightness of ${ }^{13} \mathrm{CO}(2-1)$ in the strands is still $\sim 2.5 \mathrm{~K}$, see Table 1 . 
Table 1. Results of several RADEX calculations for the filament crest using a FWHM of $0.5 \mathrm{~km} \mathrm{~s}^{-1}$, and with varying column densities, temperatures and $\left[\mathrm{H}_{2}\right] /\left[{ }^{13} \mathrm{CO}\right]$ abundance ratios (top); RADEX calculations for the strands with varying abundances for ${ }^{13} \mathrm{CO}$ and $\mathrm{C}^{18} \mathrm{O}$. The $\left[\mathrm{H}_{2}\right] /\left[{ }^{13} \mathrm{CO}\right]$ abundance ratio is varied up to a factor 3 , supported by theoretical models, and the $\left[{ }^{13} \mathrm{CO}\right] /\left[\mathrm{C}^{18} \mathrm{O}\right]$ abundance ratio is varied to obtain results consistent with the observations (bottom).

\begin{tabular}{|c|c|c|c|c|c|c|c|c|}
\hline Crest & $N_{\mathrm{H}_{2}}\left(\mathrm{~cm}^{-2}\right)$ & $T_{\text {kin }}(\mathrm{K})$ & $n_{\mathrm{H}_{2}}\left(\mathrm{~cm}^{-3}\right)$ & {$\left[\mathrm{H}_{2}\right] /\left[{ }^{13} \mathrm{CO}\right]$} & {$\left[{ }^{13} \mathrm{CO}\right] /\left[\mathrm{C}^{18} \mathrm{O}\right]$} & $T_{{ }^{13} \mathrm{CO}(2-1)}(\mathrm{K})$ & $T_{{ }^{13} \mathrm{CO}(3-2)}(\mathrm{K})$ & $T_{\mathrm{C}^{18} \mathrm{O}(2-1)}(\mathrm{K})$ \\
\hline Observed values & & & & & & 4.2 & 2.1 & 1.6 \\
\hline \multirow{6}{*}{ RADEX } & $4 \times 10^{21}$ & 10 & $10^{4}$ & $5.7 \times 10^{5}$ & 7.3 & 4.6 & 2.1 & 1.6 \\
\hline & $6 \times 10^{21}$ & 10 & $10^{4}$ & $5.7 \times 10^{5}$ & 7.3 & 4.9 & 2.6 & 2.3 \\
\hline & $6 \times 10^{21}$ & 9 & $10^{4}$ & $5.7 \times 10^{5}$ & 7.3 & 4.1 & 2.0 & 2.0 \\
\hline & $4 \times 10^{21}$ & 11 & $10^{4}$ & $8 \times 10^{5}$ & 7.3 & 4.8 & 2.2 & 1.5 \\
\hline & $4 \times 10^{21}$ & 13 & $6.5 \times 10^{3}$ & $5.7 \times 10^{5}$ & 7.3 & 6.1 & 3.0 & 1.9 \\
\hline & $3 \times 10^{21}$ & 10 & $10^{4}$ & $5.7 \times 10^{5}$ & 7.3 & 4.3 & 1.8 & 1.3 \\
\hline Strand & $N_{\mathrm{H}_{2}}\left(\mathrm{~cm}^{-2}\right)$ & $T_{\text {kin }}(\mathrm{K})$ & $n_{\mathrm{H}_{2}}\left(\mathrm{~cm}^{-3}\right)$ & {$\left[\mathrm{H}_{2}\right] /\left[{ }^{13} \mathrm{CO}\right]$} & {$\left[{ }^{13} \mathrm{CO}\right] /\left[\mathrm{C}^{18} \mathrm{O}\right]$} & $T_{{ }^{13} \mathrm{CO}(2-1)}(\mathrm{K})$ & $T_{{ }^{13} \mathrm{CO}_{(3-2)}}(\mathrm{K})$ & $T_{\mathrm{C}^{18} \mathrm{O}(2-1)}(\mathrm{K})$ \\
\hline Observed values & & & & & & 2.4 & 0.8 & 0.085 \\
\hline \multirow{9}{*}{ RADEX } & $10^{21}$ & 15 & $3 \times 10^{3}$ & $5.7 \times 10^{5}$ & 7.3 & 2.8 & 0.85 & 0.52 \\
\hline & $10^{21}$ & 15 & $2 \times 10^{3}$ & $5.7 \times 10^{5}$ & 7.3 & 2.3 & 0.59 & 0.42 \\
\hline & $10^{21}$ & 18 & $2 \times 10^{3}$ & $5.7 \times 10^{5}$ & 7.3 & 2.6 & 0.8 & 0.48 \\
\hline & $10^{21}$ & 15 & $3 \times 10^{3}$ & $3.8 \times 10^{5}$ & 7.3 & 3.7 & 1.2 & 0.78 \\
\hline & $10^{21}$ & 15 & $10^{3}$ & $3.8 \times 10^{5}$ & 7.3 & 2.0 & 0.44 & 0.39 \\
\hline & $10^{21}$ & 15 & $10^{3}$ & $2.9 \times 10^{5}$ & 7.3 & 2.4 & 0.59 & 0.52 \\
\hline & $10^{21}$ & 15 & $10^{3}$ & $1.9 \times 10^{5}$ & 7.3 & 3.0 & 0.84 & 0.74 \\
\hline & $10^{21}$ & 15 & $3 \times 10^{3}$ & $5.7 \times 10^{5}$ & 40 & 2.8 & 0.85 & 0.10 \\
\hline & $10^{21}$ & 15 & $3 \times 10^{3}$ & $5.7 \times 10^{5}$ & 50 & 2.8 & 0.85 & 0.08 \\
\hline
\end{tabular}

Notes. The predictions closely match the observed brightness towards Musca filament crest for $n_{\mathrm{H}_{2}}=10^{4} \mathrm{~cm}^{-3}$ and $T_{\mathrm{k}}=10 \mathrm{~K}$, in particular when working with $N_{\mathrm{H}_{2}}=4 \times 10^{21} \mathrm{~cm}^{-2}$ which is the column density that is associated with filament crest. The presented observed brightness is obtained from the average spectra at $N_{\mathrm{H}_{2}}>3 \times 10^{21} \mathrm{~cm}^{-2}$ (top). It demonstrates a high average $\left[{ }^{13} \mathrm{CO}\right] /\left[\mathrm{C}^{18} \mathrm{O}\right]$ abundance ratio $(\sim 50)$ in the strands. The presented observed brightness is obtained from the average spectra at $N_{\mathrm{H}_{2}}<3 \times 10^{21} \mathrm{~cm}^{-2}$. The observed brightness towards the strands generally matches predictions using $n_{\mathrm{H}_{2}}=10^{3}-3 \times 10^{3}$ and $T_{\mathrm{K}}=15-18 \mathrm{~K}$ (bottom).

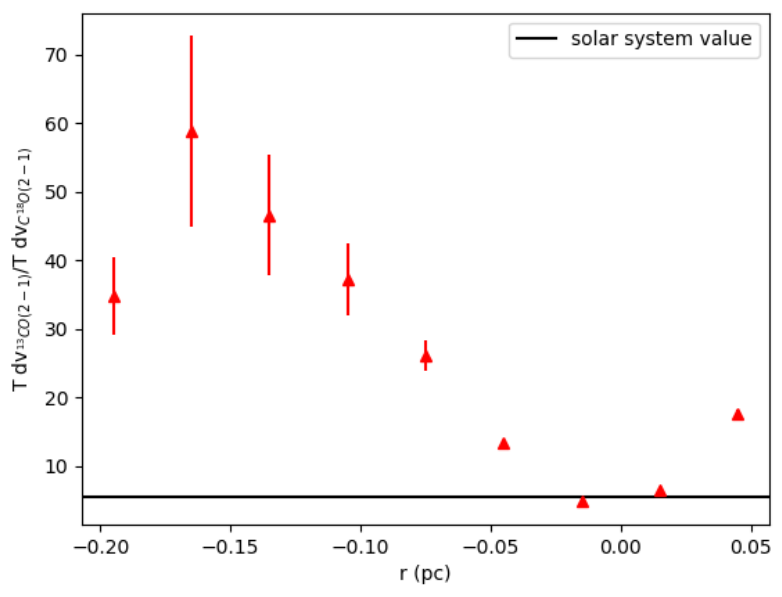

Fig. 18. Evolution of the ${ }^{13} \mathrm{CO}(2-1) / \mathrm{C}^{18} \mathrm{O}(2-1)$ integrated brightness ratio as a function of distance from the filament crest in the northern map, showing a rapid increase as a function of the distance. It shows the average ratio for the values in each distance bin of $0.03 \mathrm{pc}$ with the uncertainty related to the noise.

It was shown by Zielinsky et al. (2000) that a FUV field can create a variation of $\left[{ }^{13} \mathrm{CO}\right] /\left[\mathrm{C}^{18} \mathrm{O}\right]$ as a function of the column density due to fractionation (e.g. van Dishoeck \& Black 1988). With theoretical studies of gas exposed to a weak FUVfield $\left(\sim 1 \mathrm{G}_{0}\right)$, it was shown that carbon fractionation reactions can increase the ${ }^{13} \mathrm{CO}$ abundance up to a factor $2-3$ and thus affect the $\mathrm{CO}$ isotopologue ratios, specifically at low $A_{\mathrm{V}} \sim 1$ (e.g. Visser et al. 2009; Röllig \& Ossenkopf 2013; Szúcs et al. 2014). For $\mathrm{C}^{18} \mathrm{O}$, the wavelengths of the dissociation lines can penetrate deeper into the interior of the molecular cloud because of its poor self-shielding (e.g. Glassgold et al. 1985). Furthermore, oxygen fractionation reactions can significantly decrease the $\mathrm{C}^{18} \mathrm{O}$ abundance which can further increase the $\left[{ }^{13} \mathrm{CO}\right] /\left[\mathrm{C}^{18} \mathrm{O}\right]$ ratio. However, to this point, oxygen fractionation reactions have received little attention (Loison et al. 2019).

The observations of Musca indicate that the weak FUV field in Musca $\left(<1 \mathrm{G}_{0}\right.$ : see Paper II) might be able to significantly suppress the $\mathrm{C}^{18} \mathrm{O}$ abundance in the strands to $\left[{ }^{13} \mathrm{CO}\right] /\left[\mathrm{C}^{18} \mathrm{O}\right]=$ 40-50 on average, see Table 1 . Inside the filament crest we find $\left[{ }^{13} \mathrm{CO}\right] /\left[\mathrm{C}^{18} \mathrm{O}\right] \lesssim 10$. Significantly increasing $\left[{ }^{13} \mathrm{CO}\right] /\left[\mathrm{C}^{18} \mathrm{O}\right]$ at the crest would drop the brightness of $\mathrm{C}^{18} \mathrm{O}(2-1)$ below the observed value. This confirms that the $\left[{ }^{13} \mathrm{CO}\right] /\left[\mathrm{C}^{18} \mathrm{O}\right]$ abundance ratio increases by roughly an order of magnitude over a small physical distance $(\leq 0.2 \mathrm{pc}$ in the plane of the sky) when going from the crest to the strands at lower $A_{\mathrm{V}}$. In the study by Hacar et al. (2016) of the filament crest it was already noted that there were some indications of an increase of $\left[{ }^{13} \mathrm{CO}\right] /\left[\mathrm{C}^{18} \mathrm{O}\right]$ towards lower $A_{V}(<3)$ areas of the filament crest. Including the strands into the analysis, we now demonstrate that there is indeed a strong drop of $\mathrm{C}^{18} \mathrm{O}$ abundance for low $A_{\mathrm{V}}$ gas in the Musca cloud. This observation indicates that even a weak FUV field can have a large impact on the molecular cloud chemistry of CO. The 
formation of the dense filament crest is thus necessary for sufficient shielding such that $\mathrm{C}^{18} \mathrm{O}$ can form and implies that $\mathrm{C}^{18} \mathrm{O}$ is not a good column density tracer.

\section{Discussion}

\subsection{A cylindrical geometry for the Musca filament crest and strands}

In Sect. 5, from ${ }^{13} \mathrm{CO}$ line ratios, we derived estimates of the densities for the emitting $\mathrm{CO}$ gas in a section of the Musca filament to be $n_{\mathrm{H}_{2}}=1-3$ and $6-13 \times 10^{3} \mathrm{~cm}^{-3}$ in the strands and crest respectively. Using the Herschel total column densities towards the same directions (crest and strands), and assuming the standard $\mathrm{CO}$ abundance and gas/dust ratio, we then obtained the typical sizes on the line of sight for the crest and strands: $0.1-0.2 \mathrm{pc}$ for the crest and $0.2-0.5 \mathrm{pc}$ for the strands. This is very similar to their respective sizes projected on the sky. This clearly shows that both the strands and the crest are not more elongated along the line of sight than their transverse size in the plane of sky. Our CO observations therefore confirm that the Musca crest and strands correspond more to a filament, that is a cylindrical structure, than to a sheet seen edge-on as proposed in Tritsis \& Tassis (2018). The $0.1-0.2 \mathrm{pc}$ line of sight size of the crest is clearly in disagreement with the proposed $6 \mathrm{pc}$ size in Tritsis \& Tassis (2018) to explain the regular pattern of striations observed in the Musca cloud with MHD waves

Looking carefully at the Herschel maps in Cox et al. (2016) we actually see that the striations are not necessary originating from the crest of the filament as assumed in Tritsis \& Tassis (2018). The striations are indeed seen over an extended region of $\sim 2 \times 4 \mathrm{pc}$ around the crest (see Fig. 2 in Cox et al. 2016), that we here refer to as the ambient cloud. It could thus come from some extended, more diffuse gas around the filament.

For the immediate surroundings of the crest seen in our APEX maps, we also get a density from $\mathrm{CO}$ which points to sizes along the line of sight not larger than $\sim 0.5 \mathrm{pc}$ only. This is still far from the $6 \mathrm{pc}$ required to explain the striations with the magnetohydrodynamic waves. On the other hand our APEX CO study is limited to only the most nearby regions of the crest, of the order of $\sim 0.2 \mathrm{pc}$. At larger scale, the ambient cloud surrounding the filament could have a line of sight size up to $6 \mathrm{pc}$, and could then host the striation pattern. Using the typical observed column density of $N_{\mathrm{H}_{2}}=1-3 \times 10^{21} \mathrm{~cm}^{-2}$ in the direction of the strands, and assuming all this column density could be due to the surrounding ambient cloud, we would get for a line of sight length of $6 \mathrm{pc}$ a typical density for this medium as low as $\sim 50-150 \mathrm{~cm}^{-3}$. Such low densities might actually not be visible in $\mathrm{CO}$ and may correspond to some surrounding $\mathrm{CO}$ dark gas.

In conclusion, our $\mathrm{CO}$ observations clearly show that the densest region (crest and strands) of Musca is mostly a cylindrical filamentary structure. The striations are either not related to the proposed MHD vibrational modes of Tritsis \& Tassis (2018) or these modes, if they require a depth of $6 \mathrm{pc}$, have developed in the extended ambient, probably CO-dark low density medium $\left(\sim 50-150 \mathrm{~cm}^{-3}\right)$ surrounding the Musca filament.

\subsection{Continuous mass accretion on the filament crest in Musca}

The velocity structure of the Musca filament crest, displayed in Fig. 9 and that of the surrounding strands, traced with ${ }^{13} \mathrm{CO}(2-$ 1), clearly indicates that in both APEX maps velocity gradients from blue-shifted extended gas to red-shifted dense gas are monotonic. These continuous gradients are a hint of slowing down of the large scale gas reaching the crest, and then point to accretion.

Interestingly enough these monotonic gradients have an opposite direction at both observed locations, see Fig. 9, similar to what was observed for instance in the massive DR21 ridge (Schneider et al. 2010). In DR21 this behaviour was proposed to be the result of a global collapse with inflowing subfilaments driving the velocity gradients.

Studying the location and velocity of the strands in the observed APEX maps, we found that the strands have changed side of the filament from our point of view, see Fig. 14, while at both locations the velocity of the strands are blueshifted, see e.g. Fig. 11. The blueshifted part of the filament crest velocity field, traced by $\mathrm{C}^{18} \mathrm{O}(2-1)$, is always located at the side where the blueshifted strands connect with the filament crest, which strongly suggests a link between the velocity gradient over the filament crest and the immediate surrounding blueshifted strands. These observations point to a scenario where the strands are being accreted on the crest. As it is possible that this mass accretion is not exactly fixed on the filament axis, it can also deposit angular momentum inside the filament which could contribute to the $\mathrm{C}^{18} \mathrm{O}(2-1)$ crest velocity field. This is schematically shown in Fig. 19.

Velocity gradients perpendicular to filaments have been found in other clouds as well, e.g.: DR21 (Schneider et al. 2010), IRDC 18223 (Beuther et al. 2015), Serpens (Dhabal et al. 2018; Chen et al. 2020) and SDC13 (Williams et al. 2018). In these studies, the observed velocity gradients have also been found to be possible indications of mass accretion by the filament from inflowing lower-density gas. However, the interpretation is often complicated by e.g. the presence of multiple velocity components (e.g Schneider et al. 2010; Beuther et al. 2015; Dhabal et al. 2018). In the southern map of Musca there is a hint of a second component in $\mathrm{C}^{18} \mathrm{O}$, but in the northern map the emission only shows a single velocity component which experiences the velocity gradient. This strongly suggests that the velocity gradient in Musca is indeed a mass accretion signature.

\subsection{A potential H I cloud-cloud collision scenario to form the Chamaeleon-Musca complex}

We obtain a scenario where the dense Musca filament is continuously accreting mass from large scale inflow, which fits with observed indications of filament accretion shocks towards the Musca filament in Paper II. Here we study the large-scale kinematics of the Chamaeleon-Musca complex to constrain the mechanism that drives the continuous mass accretion of the Musca filament and thus is responsible for the formation of the Musca filament.

\subsubsection{A $50-100 \mathrm{pc}$ coherent cloud complex in $\mathrm{CO}$ and $\mathrm{H}$}

The results of the NANTEN CO survey of the ChamaeleonMusca region in Mizuno et al. (2001) show the existence of a well-defined velocity $\left(-1\right.$ to $\left.6 \mathrm{~km} \mathrm{~s}^{-1}\right)$ and spatially coherent $\mathrm{CO}$ complex extending over roughly $20^{\circ} \times 24^{\circ}$, i.e. $35-40 \mathrm{pc}$ at $90 \mathrm{pc}$ to $70-80 \mathrm{pc}$ at $190 \mathrm{pc}$ while the analysis of Gaia data towards the CO clouds Cha I, II, III and Musca indicates walls of extinction ranging from 90 to $190 \mathrm{pc}$ along the line of sight. This suggests that the global CO gas and the well known clouds Cha I, II, III and Musca are parts of a single complex of size 50 to $100 \mathrm{pc}$ in projection and along the line of sight. 
Northern map

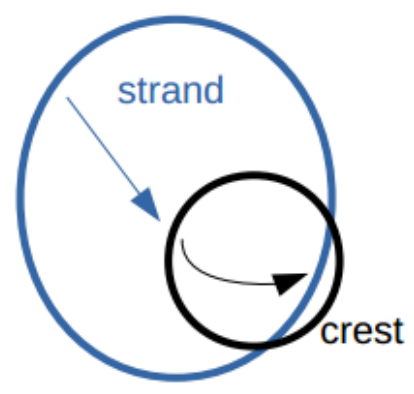

Southern map

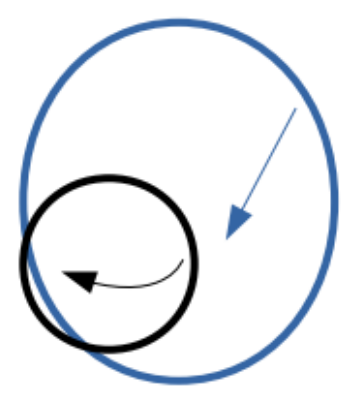

\section{our point of view}

Fig. 19. Schematic illustration of the proposed accretion scenario responsible for the velocity gradient over the crest, possibly also related to some angular momentum deposition due to an accretion impact parameter, as seen from above the filament. The blue arrow indicates the motion of the strands compared to the crest from our point of view and the black arrows indicate the possible velocity field over the crest which is responsible for the observed velocity gradient.

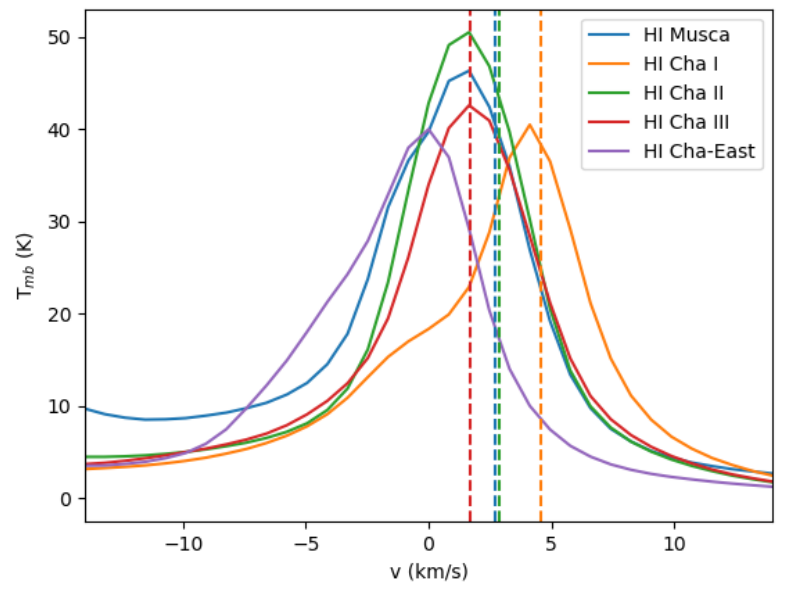

Fig. 20. H I spectra averaged towards Cha I, Cha II, Cha III, the eastern region of the Chamaeleon cloud and Musca. The dashed vertical lines give the ${ }^{12} \mathrm{CO}$ velocities of the dense regions Cha I, Cha II, Cha III and Musca (Mizuno et al. 2001). This shows that the dense gas traced by ${ }^{12} \mathrm{CO}$ for all regions is redshifted compared to their $\mathrm{HI}$ emission, identical to what is found for the filament crest of Musca when comparing ${ }^{12} \mathrm{CO}$ and $\mathrm{C}^{18} \mathrm{O}$.

To go one step further, we investigated the atomic hydrogen (H I $21 \mathrm{~cm}$ line from the Galactic All Sky Survey (GASS) with the Parkes telescope (McClure-Griffiths et al. 2009; Kalberla et al. 2010; Kalberla \& Haud 2015) towards the ChamaeleonMusca region. The data has a spectral resolution of $0.8 \mathrm{~km} \mathrm{~s}^{-1}$ and a spatial resolution of $16^{\prime}(\sim 0.65 \mathrm{pc})$. This does not resolve $\mathrm{CO}$ clouds individually, but we find in Fig. 20 that the $\mathrm{H}$ I spectra have central velocities ranging from -2 to $5 \mathrm{~km} \mathrm{~s}^{-1}$ for the CO clouds. In Fig. 21 we show in the upper map the H I integrated emission between -10 and $10 \mathrm{~km} \mathrm{~s}^{-1}$ with contours of the Planck brightness at $353 \mathrm{GHz}$ which indicates that there is a good spatial coincidence between $\mathrm{H}_{\mathrm{I}}$ and extended dust emission for the Chamaeleon-Musca complex. Fitting a gaussian to the H I spectra, we obtain a velocity field which is mapped in the lower map of Fig. 21. It shows that the whole complex displays a global velocity gradient from (slightly south) east at $-2 \mathrm{~km} \mathrm{~s}^{-1}$ to (slightly north) west at $+5 \mathrm{~km} \mathrm{~s}^{-1}$ which corresponds well to a similar ${ }^{12} \mathrm{CO}$ east-west gradient along a filamentary feature $\sim 15^{\circ}$ long (i.e. $37 \mathrm{pc}$ long in projection at $140 \mathrm{pc}$; Mizuno et al. 2001) which is also seen in Planck dust emission. We note that the magnitude of this gradient is similar to what was found for giant molecular clouds in M33 and M51, for which it had been proposed that it would be related to galactic proand retrograde rotational motion (Braine et al. 2018, 2020). The global velocity field in the Chamaeleon-Musca complex might thus also be linked to some galactic rotation. However, here it would then be retrograde motion unlike clouds in M33 and M51 which are mostly found to be prograde. While the velocity gradient in $\mathrm{H}$ I over the region is roughly identical to that of $\mathrm{CO}$, the $\mathrm{H}_{\mathrm{I}}$ linewidths of $\sim 5-6 \mathrm{~km} \mathrm{~s}^{-1}$ are slightly larger than the linewidths in CO (2 to $2.8 \mathrm{~km} \mathrm{~s}^{-1}$ in Mizuno et al. 2001), as expected if the turbulent motions in $\mathrm{HI}$ are driving the $\mathrm{CO}$ motions as proposed for instance in numerical simulations of cloud formation by Koyama \& Inutsuka (2002).

It is therefore clear that a coherent cloud complex exists connecting all known CO clouds of the region (Cha I, II, II and Musca) as well as a few additional more diffuse $\mathrm{CO}$ clouds such as Cha East and smaller $\mathrm{CO}$ clouds discussed for instance in Mizuno et al. (1998).

\subsubsection{Dense gas mostly red-shifted in Chamaeleon/Musca}

Studying the CO isotopologues towards Musca, we found that the dense filament crest, traced by $\mathrm{C}^{18} \mathrm{O}$, is at redshifted velocities compared to the molecular ambient cloud traced by ${ }^{12} \mathrm{CO}$ (Sect. 4.4). With $\mathrm{HI}_{\mathrm{I}}$, it is possible to study the kinematics of the even lower density and larger scale ambient gas. Inspecting the average H I spectrum towards the Musca cloud in Fig. 20, we found that the NANTEN $2{ }^{12} \mathrm{CO}$ emission also appears redshifted compared to $\mathrm{H}_{\mathrm{I}}$ in the same way as $\mathrm{C}^{18} \mathrm{O}$ compared to ${ }^{12} \mathrm{CO}$ at smaller scale in Fig. 8. This demonstrates that denser gas in the Musca cloud is systematically redshifted from low density, large scale atomic and molecular gas down to the Musca strands and filament crest.

In Fig. 21, we plot the individual $\mathrm{H}$ I spectra (in red) of the Chamaeleon/Musca clouds (Cha I, Cha II, Cha III, Cha East and Musca) together with their ${ }^{12} \mathrm{CO}$ velocities reported in Mizuno et al. (2001) (dashed lines) in comparison with the globally averaged $\mathrm{H}_{\text {I }}$ spectrum (in blue). We see that while the

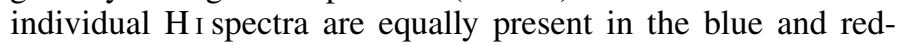
shifted parts of the average $\mathrm{H}$ i spectrum, the $\mathrm{CO}$ gas tends to be mostly in the red-shifted part and is basically always red-shifted compared to the individual $\mathrm{H}$ I spectra towards each regions. This suggests that the velocity asymmetry of the dense gas, being mostly red-shifted, seen towards Musca is a general trend for the whole Chamaeleon-Musca cloud complex. Combining this with the identical redshifted asymmetry at small scales for Musca suggests a dynamical coherence in the complex, i.e. similar kinematic asymmetry, from the Hicloud with a size of $\sim 50-100 \mathrm{pc}$ down to $\sim 0.1 \mathrm{pc}$ around the Musca filament crest. This puts forward a scenario where the mass accretion of the dense (star forming) gas, which was in particular proposed based on the velocity gradients over the filament crest, in the complex is directly related to this large scale, and asymmetric $\mathrm{HI}_{\mathrm{I}} / \mathrm{CO}$ kinematics of the full complex.

We conclude that the local kinematic asymmetry observed towards Musca is observed for the whole complex and 

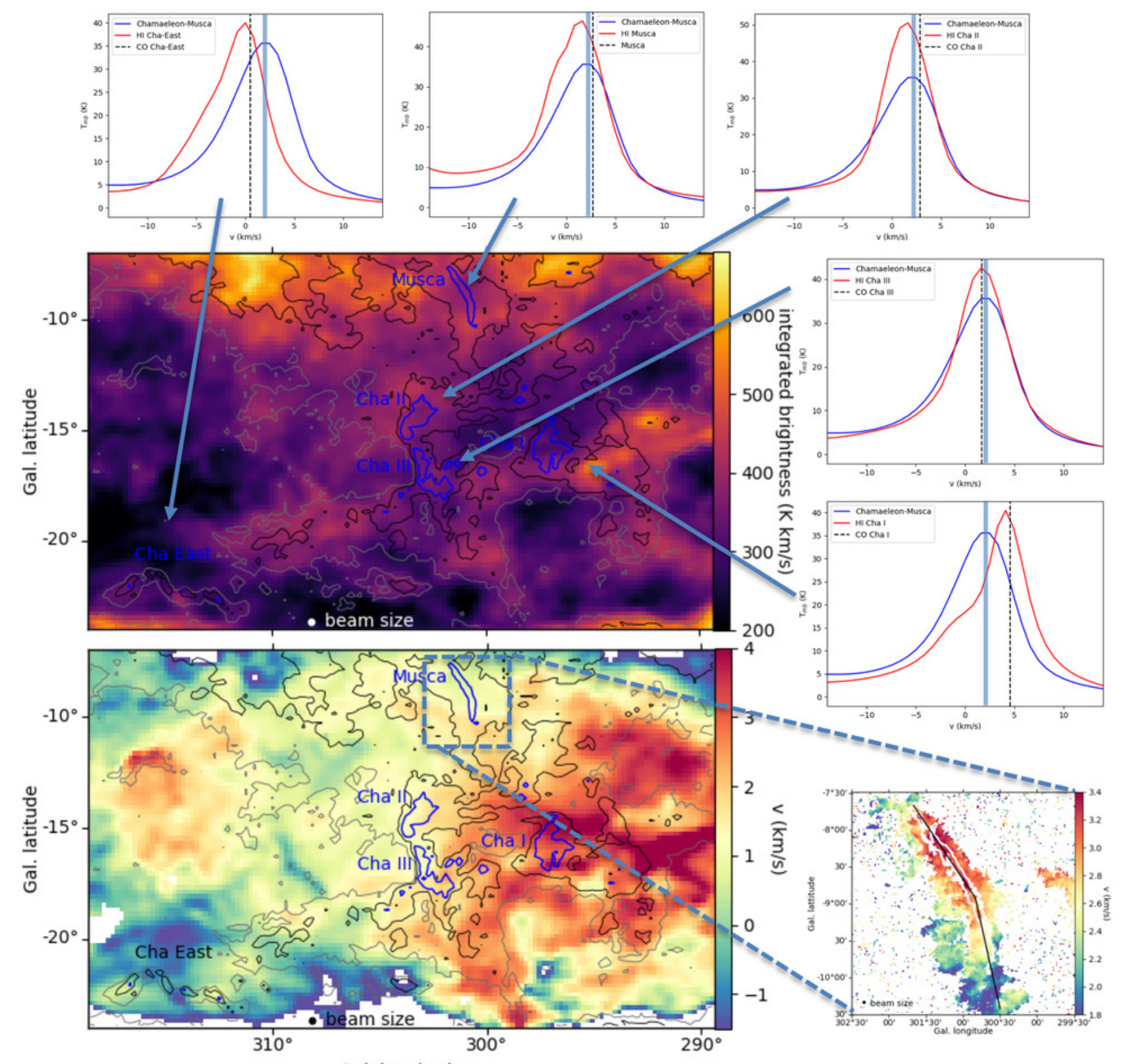

Gal. longitude

Fig. 21. Integrated H I map (top) and the velocity field (below) of the Chamaeleon-Musca complex from fitting a single gaussian to the H I spectra, with overplotted Planck brightness contours at $353 \mathrm{GHz}$. The blue contours highlight the dense Chamaeleon molecular clouds and the Musca filament, while the black and grey contours indicate the more extended continuum emission from Planck. Around the two maps, the average $\mathrm{H}$ I spectrum of the full complex and its peak emission (blue) is compared with the local Hi spectrum (red) and velocity of the CO gas (black dashed line) (Mizuno et al. 2001) from the selected dense region (Cha I, Cha II, Cha III, Cha East and Musca). In the lower right figure, the ${ }^{12} \mathrm{CO}$ velocity field from the NANTEN2 data is shown.

individually for each CO cloud of the complex. This suggests that the region corresponds to a single event of cloud formation. We argue in the following subsection that a $\mathrm{H}$ i cloud-cloud collision event can explain the kinematic asymmetry, which can fit with the observed indications of more than one velocity component in several $\mathrm{H}$ i spectra.

\subsection{Asymmetric inflow guided by the magnetic field in a H I cloud-cloud collision}

The asymmetry in velocity between low and high density gas at all scales is difficult to explain with a classical view of an isotropic injection of turbulence at large scales. In this view, the velocity streams would be on average equally blue and redshifted. In contrast, any scenario based on a $\left(\mathrm{H}_{\mathrm{I}}\right)$ cloud-cloud collision can easily introduce asymmetries if the two clouds in collision have different initial properties and substructure, which is actually expected and natural. In the case of a ( $\left.\mathrm{H}_{\mathrm{I}}\right)$ cloud-cloud collision with some primordial density substructure, numerical simulations show that such a collision is prone to concentrate the pre-existent structures (e.g. Inoue \& Inutsuka 2012; Inoue \& Fukui 2013; Inoue et al. 2018; Iwasaki et al. 2019). This concentration of matter can originate from a locally curved magnetic field leading to dense structures perpendicular to the 
(a)
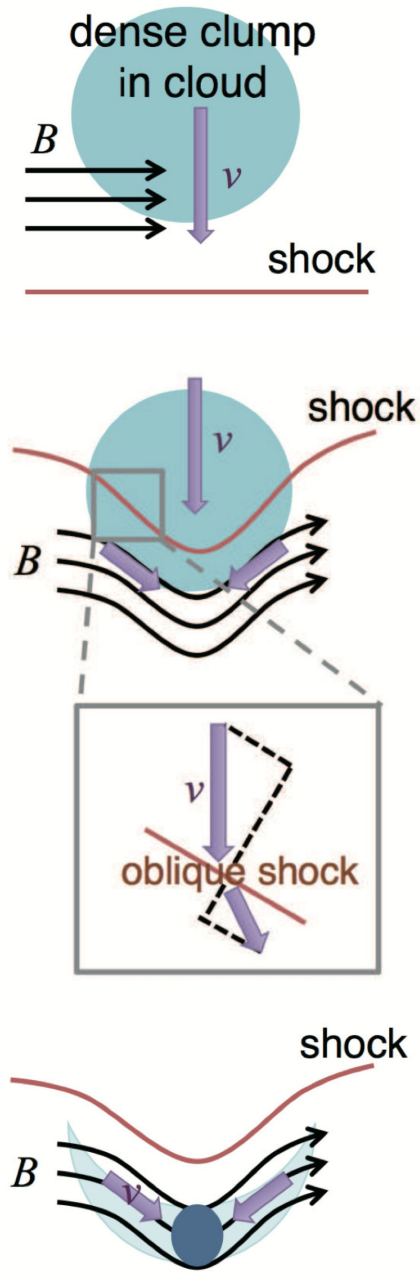

Shock asymmetry (b)

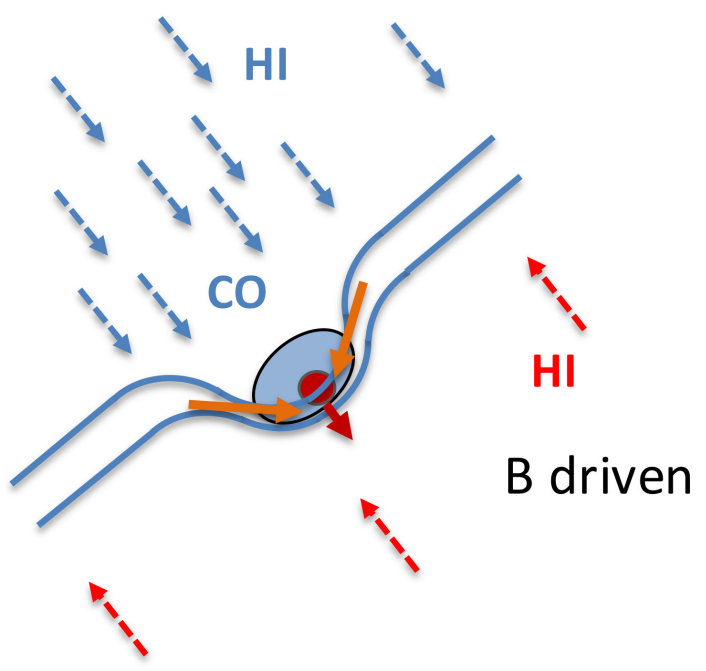

(c)

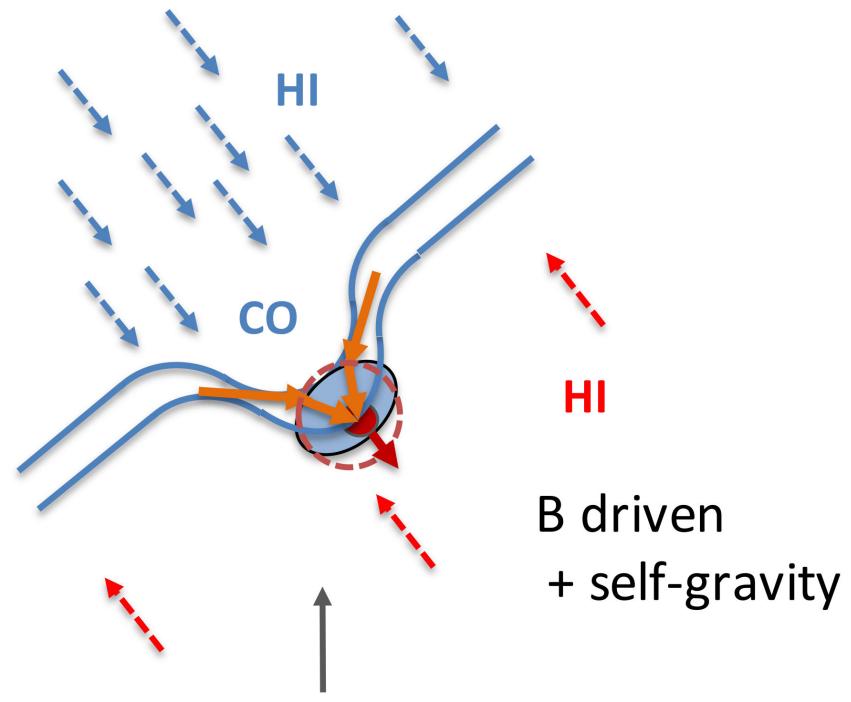<smiles>[Y]</smiles>

Fig. 22. Proposed sketch, observed from above the Musca filament, to explain the spatial and kinematic asymmetries of Musca originating from a $\mathrm{H}$ I cloud-cloud collision event (blue and red dashed arrows) following the Inoue scenario (see panel $a$, Inoue et al. 2018). The asymmetry between $\mathrm{CO}$ and $\mathrm{H}$ i could be due to the effect of compression of a clump in a cloud-cloud collision. The resulting bent of the magnetic field (blue lines) drives the gas towards the apex along the magnetic field, leading to a concentration of the mostly blue-shifted but slowed down (i.e. becoming red-shifted compared to the local mostly blue-shifted $\mathrm{H}$ I gas) $\mathrm{CO}$ gas (B driven concentration, panel b). Later on, the self-gravity of the strong concentration of matter (filament crest) can curve the concentration flows (see panel c) continuing the accretion onto the crest while magnetic field slowly drifts through the crest. The crest would then be in equilibrium between an asymmetric accretion and the tension of magnetic field. The progressive slowing down of the accretion flow, observed in the strands, from the bluest gas to the crest velocity could be partly due to magnetic pressure (the flows get less aligned with the magnetic field as self-gravity starts to take over) and perhaps some contribution from the turbulent pressure as it gets close to the crest (see Sect. 6.6). Seen from the side, the magnetic field would naturally appear perpendicular to the crest of the filament as observed.

magnetic field. This would fit with the generally accepted view that inflows towards the dense filaments are guided by the magnetic field (e.g. Banerjee et al. 2009; Soler et al. 2013), possibly in the form of striations (Palmeirim et al. 2013; Cox et al. 2016). This (Inoue) mechanism is based on the fact that when a shock wave impacts a slightly denser structure, the local magnetic field is bent around the structure and then channels the streams of gas (through an oblique shock; see Fig. 22a) towards the apex of the bent which is then rapidly the densest part of the original structure (Inoue \& Fukui 2013; Vaidya et al. 2013).

Interestingly enough such a bent of the magnetic field by a shock in the Inoue mechanism naturally predicts a spatial and kinematic asymmetry of the dense structure with the densest region, the filament crest, being concentrated on the front side of the original structure. Figure 22 illustrates how such a scenario could explain the described kinematical asymmetry of Musca as well as the indicated spatial asymmetry in Sect. 4.4. The Musca filament/crest would trace this front side of the original structure which has been concentrated by favoured channelling parallel to the bent magnetic field (see the sketch in the right panels of Fig. 22). This dense structure is also the most slowed down gas by the opposite stream of gas from the red-shifted H i cloud (see panel b of Fig. 22). It would explain why all dense CO structures in the region appear red-shifted as they would be the most 


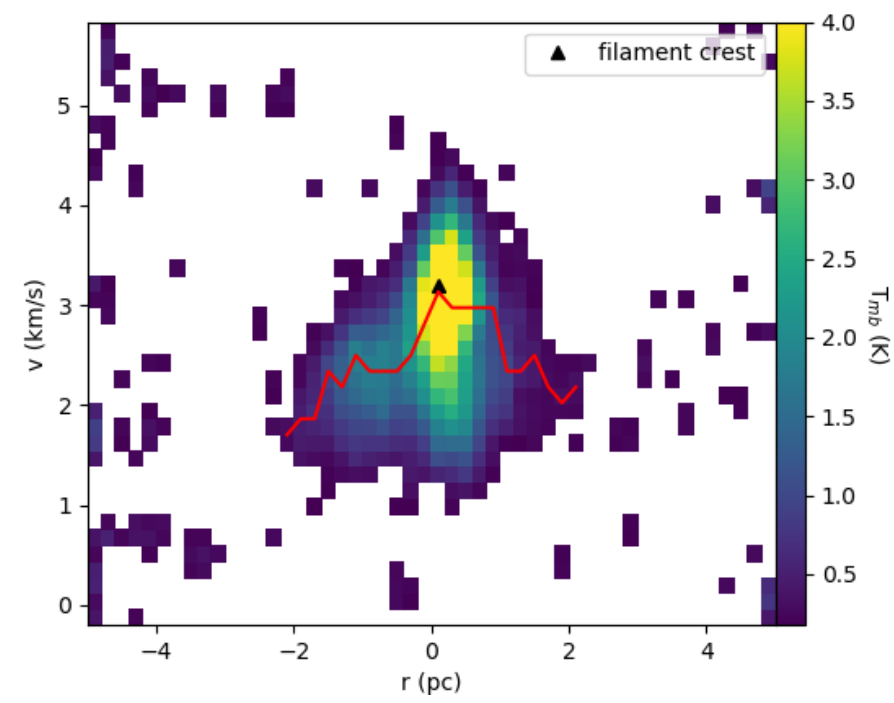

Fig. 23. PV diagram as a function of the distance from the center of the Musca filament for the ${ }^{12} \mathrm{CO}(1-0)$ emission from the NANTEN2 data. The red line follows the velocity with maximal brightness as a function of the distance from the filament crest. The PV diagram shows the expected V-shape from the Inoue scenario with respect to the velocity of the dense filament crest (Hacar et al. 2016) which is at the redshifted apex of the V-shape.

sensitive to the dragging force of the opposite flow of gas from the colliding red-shifted $\mathrm{H}_{\mathrm{I}}$ cloud. If globally $\mathrm{H}_{\mathrm{I}}$ is dominated by the blue-shifted Higas, this would explain the tendency to have the $\mathrm{H}$ i spectrum slightly blue-shifted compared to the $\mathrm{CO}$ gas of all molecular clouds in the $\mathrm{Cha} / \mathrm{Musca}$ region. In this scenario the magnetic field would channel the pre-existent mostly blue-shifted structure and its immediate surroundings, while slightly slowing down and becoming more red-shifted and therefore allowing for more accretion of faster blue-shifted gas from behind. The orientation of this organised inflow by two intersecting sheet-like structures could then explain why most $\mathrm{CO}$ emission is observed east of the filament. This scenario has the advantage to explain both the spatial and velocity asymmetries, and to explain the fact that the magnetic field is perpendicular to the filament/crest.

In particular, this bending of the magnetic field would lead to an observable signature in the PV diagram of the Musca cloud. This signature is displayed in Fig. 22 and in Fig. 16 from Arzoumanian et al. (2018). Specifically, the kinematic signature consists of a "V-shape" for the inflowing molecular cloud with the densest gas at the apex of this V-shape. A V-shape in the PV diagram was reported by Arzoumanian et al. (2018) for a filament in the Taurus molecular cloud. Figure 23 shows the PV diagram of the Musca cloud from the NANTEN2 data. This PV diagram shows exactly such a $\mathrm{V}$-shape, with the $\mathrm{C}^{18} \mathrm{O}$ velocity of the filament crest at the apex of this V-shape.

\subsection{Inhomogeneities in the inflow driven by local gravity or large-scale dynamics}

While the Inoue scenario may well explain the observed local asymmetries towards the Musca filament, the change of direction for the velocity gradient along the filament discussed in Sect. 6.2 might be more difficult to explain with this scenario alone. We note however that numerical simulations such as the ones presented in Clarke et al. $(2017,2018)$ reproduce very well this behaviour (see also simulations presented in Schneider et al.

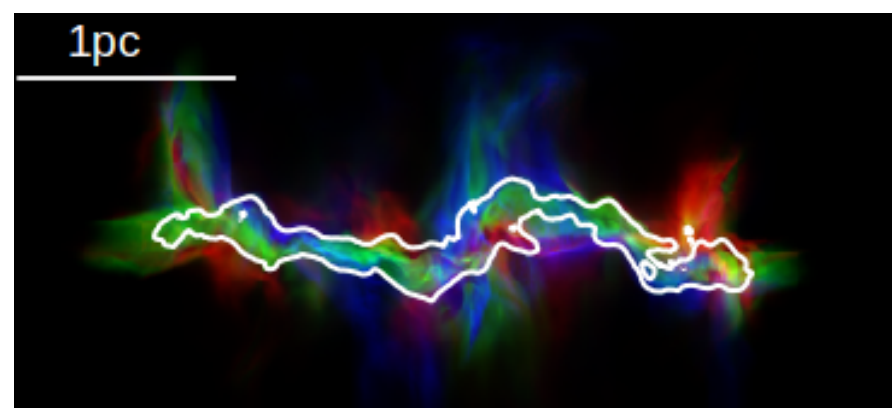

Fig. 24. RGB image using ${ }^{12} \mathrm{CO}(1-0)$ synthetic observations (red: $0.5-2 \mathrm{~km} \mathrm{~s}^{-1}$ \& blue: -2 to $-0.5 \mathrm{~km} \mathrm{~s}^{-1}$ ) and ${ }^{13} \mathrm{CO}(1-0)$ synthetic observations (green: -0.5 to $0.5 \mathrm{~km} \mathrm{~s}^{-1}$ ) of the simulations in Clarke et al. (2018). The white contour encloses the region with integrated $\mathrm{C}^{18} \mathrm{O}(1-0)$ brightness $>1 \mathrm{~K} \mathrm{~km} \mathrm{~s}^{-1}$. This shows that close to the filament the direction of the velocity gradient can change, similar to what is observed in Musca, as a result of turbulent motions.

2010 to explain similar features in the DR21 filament/ridge). These simulations consist of a cylindrical converging flow which produces an accreting dense filament. Turbulence in the flow leads to inhomogeneities in the accretion and substructures in the filament with accretion flows which are not always landing on the filament from the same side of the crest. The simulation includes hydrodynamics, self-gravity, a weak FUV-field $\left(1.7 \mathrm{G}_{0}\right)$, and heating and cooling coupled with non-equilibrium chemistry. This allows for the self-consistent formation of $\mathrm{CO}$ and thus the production of synthetic $\mathrm{CO}$ observations as shown in Fig. 24, which present an RGB image from the simulation that highlights the locations of the blue- and redshifted mass reservoir around the dense filament. These synthetic observations indicate that there is no clearly separated blue- and redshifted mass reservoir close to the filament crest, but rather that the location of the dominant inflowing mass reservoir alternates to each side of the filament as a consequence of the turbulence in the inflowing mass reservoir (Clarke et al. 2017, Fig. 24). The simulations demonstrate that in a cylindrical area close to the filament there can be large local position variations of the converging flows towards the filament. In Musca this relatively cylindrical area has a size of the order of $0.4 \mathrm{pc}$, covering the filament crest and strands.

We conclude that these inhomogeneities in the inflow can be expected at least in a non-magnetic case (the above discussed simulations do not include magnetic field). It is not clear though what is the main driver of these local variations of the converging flows in Musca. Including the possible role of magnetic field, it can either originate purely from the original kinematic fluctuations from large scales, forcing the magnetic field guidance or could be partly due to self-gravity close to the crest which could decouple the gas inflow from the guidance of magnetic field allowing for these variations along the filament.

\subsection{A two step scenario: $B$ driven followed by a gravity driven and $B$ regulated accretion}

As shown in the previous section thanks to numerical simulations, self-gravity of the filamentary structure may explain the changing direction of the velocity gradients along the crest of the filament. We may then have a situation as shown in panel $\mathrm{c}$ of Fig. 22 where there is a transition from the B (magnetic field) driven concentration to a gravity driven accretion regime close to the crest. This may correspond to the region of the strands 
where we observe a smooth ${ }^{13} \mathrm{CO}$ velocity gradient from 3.1 to $3.5 \mathrm{~km} \mathrm{~s}^{-1}$ and from 2.7 to $3.1 \mathrm{~km} \mathrm{~s}^{-1}$ for the northern and southern APEX maps, respectively. The progressive slowing down of the blue-shifted gas to the velocity of the crest could then be due to the need to cross some magnetic field lines to reach the crest. Indeed, close to the crest where the self-gravity of the crest is maximal, the magnetic field should slowly drift through the filament crest in the Inoue scenario (Fig. 22) as it is dragged by the larger scale pressure from the red-shifted gas. So we would have a scenario where the gas is accelerated by gravity but slowed down by magnetic pressure to reach the up-stream crest.

Alternatively in Shimajiri et al. (2019) for the B211/3 filament in Taurus, gravity was claimed to accelerate gas from large to small scales, and with a slowing down of velocities close to the crest being due to a large turbulent pressure (up to an order of magnitude larger than the thermal pressure). To obtain a fit to the data, they had to assume that the whole CO linewidth would represent an effective turbulent pressure. In Musca in the APEX and NANTEN2 maps we see that the global velocity dispersion appears to be dominated by bulk motions with velocity gradients, and not by isotropic turbulence. We easily expect that at higher spatial resolution an even larger fraction of the linewidth is due to bulk motions rather than to isotropic turbulence. Also we note that the velocity dispersion (expressed in $\sigma$ ) is much smaller in Musca compared to B211/3. Instead of $0.9 \mathrm{~km} \mathrm{~s}^{-1}$ for B211/3, we obtain a maximal $\sigma=0.4-0.5 \mathrm{~km} \mathrm{~s}^{-1}$ over the whole region for Musca in the NANTEN2 ${ }^{12} \mathrm{CO}$ data (e.g. Fig. 13), and even down to $\sigma \sim 0.2 \mathrm{~km} \mathrm{~s}^{-1}$ close to the crest in the ${ }^{13} \mathrm{CO}$ APEX data. The possible deceleration thanks to a possible contribution of the turbulent motions to pressure in the immediate surroundings of the crest is therefore reduced in Musca compared to B211. Since the gravitational acceleration in Musca is also reduced (lower mass filament) it is not possible to firmly reject some effect of the turbulent pressure effect, but we point out here that our scenario can explain the observed deceleration without heavily relying on an hypothetical full conversion of $\mathrm{CO}$ linewidth into effective sound speed such as in Shimajiri et al. (2019) for B211/3. Altogether we may actually have a mixture of magnetic and turbulent pressure to explain the deceleration of the accretion flow onto the crest.

We finally note that our scenario might also explain the geometry and velocity field of the B211/3 filament. This filament shows an asymmetry between the blue- and redshifted sides of the accretion flow, and also displays a V-like shape in the PV diagram (Shimajiri et al. 2019). The proposed angle of $130^{\circ}$ between the two sheets in Shimajiri et al. (2019) could actually trace the bent of the magnetic field in the Inoue scenario.

\subsection{The physical scale of dominant self-gravity}

Here we compare the typical observed relative motions between the crest and the surrounding gas with the expected self-gravity velocities to discuss at which scale gravity may be responsible for the observed relative motions. In Chen et al. (2020) it is proposed to use the non-dimensional parameter $\mathrm{C}_{v}$ to differentiate between gravity-driven mass inflow and other sources of motions (shock compression in Chen et al. 2020), with $\mathrm{C}_{v}$ expressed as

$C_{v}=\frac{\Delta v_{\mathrm{h}}^{2}}{G M(r) / L}$

where $\Delta v_{\mathrm{h}}$ is half of the velocity difference over the filament and $M(r) / L$ the mass per unit length at the distance $r$ from the center of the crest.
On large scales, the velocity difference between the filament and the CO gas reaches values of the order of $1 \mathrm{~km} \mathrm{~s}^{-1}$ at both sides of the filament crest, see Fig. 23. With a mass per unit length of $15.6 M_{\odot} \mathrm{pc}^{-1}\left(22.3 M_{\odot} \mathrm{pc}^{-1}\right.$ corrected to $\left.d=140 \mathrm{pc}\right)$ for the Musca filament, this corresponds to $C_{v}=15$. At the pc scale, the kinematics are thus not dominated by the self-gravity of the filament. In other words the actual crossing time is clearly smaller than the free-fall time from the self-gravity of the filament. At the $0.4 \mathrm{pc}$ scale of the strands, we still have a velocity difference of $\sim 0.4 \mathrm{~km} \mathrm{~s}^{-1}$ leading to $C_{v}=2.4$. This suggests that self-gravity may start to play a role but is not dominant yet. At the scale of the filament crest with a velocity difference of $0.2 \mathrm{~km} \mathrm{~s}^{-1}$ we obtain $C_{v}=1.1$. We thus find that gravity may indeed take over from the large scale motions at sub-pc scales, reinforcing our proposed scenario in two steps with a B-driven followed by a gravity driven accretion when matter is reaching the crest of the filament at sub-pc scale.

When we apply the $C_{v}$ criterion to study the importance of self-gravity for the B211/3 filament, we similarly found large values of $C_{v}$ at the pc scale, and at the sub-pc scale close to the crest that $C_{v}$ could be approaching a value of 1 . The velocities of the large scale flows in B211/3 are found to be typically 2-3 times larger than in Musca at the same distances from the crest while the $M / L$ value is roughly 3 times larger $\left(54 M_{\odot} \mathrm{pc}^{-1}\right)$. $C_{v}$ is therefore found to be typically 2-3 times larger in B211/3 at large scales.

\subsection{Future star formation in the Musca filament}

In this paper we proposed that a large scale colliding $\mathrm{H}$ i flow, forming the Chamaeleon-Musca complex, could be at the origin of the proposed mass accretion on the Musca filament crest. Here, we investigate whether such continuous mass accretion can eventually lead to star formation in the Musca filament.

In the NANTEN2 data, most molecular gas traced by ${ }^{12} \mathrm{CO}$ is present in the filament and in a structure east of the filament which has a relative velocity of -0.5 to $-1 \mathrm{~km} \mathrm{~s}^{-1}$ compared to the velocity of the filament. Using simple assumptions, we now estimate a time scale for accretion of this gas on the filament. We assume a projected inflow velocity in the plane of the sky of $0.7 \mathrm{~km} \mathrm{~s}^{-1}$ towards the filament, while the distance perpendicular to the crest of this ${ }^{12} \mathrm{CO}$ emission is generally of the order of 0.5 to $1 \mathrm{pc}$. It would thus require roughly $0.7-1.4 \mathrm{Myr}$ for the nearby ambient cloud within this radius to be accreted on the filament/strands. Then there are the strands at a velocity of -0.4 to $-0.5 \mathrm{~km} \mathrm{~s}^{-1}$ along the line of sight compared to the filament crest. These strands are present from the edge of the filament crest up to a distance of $\sim 0.4 \mathrm{pc}$. At this velocity, the accretion of these strands on the filament crest would take close to $1 \mathrm{Myr}$. The filament crest has a velocity gradient, with a maximal velocity difference of $0.15-0.2 \mathrm{~km} \mathrm{~s}^{-1}$. This results in a similar crossing timescale for the filament crest of 0.7 Myr. However, one should note that this velocity gradient is not necessarily related to dispersion of the filament, and that the increasing role of gravity will confine the filament such that this timescale is a lower limit.

Similar to Palmeirim et al. (2013) we can estimate the mass accretion rate per unit length on the filament at $R=0.4 \mathrm{pc}$. For this we need a density estimate at $R=0.4 \mathrm{pc}$. Here we use the same approach as Palmeirim et al. (2013) by extracting the density from the fitted Plummer model to the averaged Musca filament (Cox et al. 2016). This results in a mass accretion estimate of $14 M_{\odot} \mathrm{pc}^{-1} \mathrm{Myr}^{-1}$. With this mass accretion rate it would roughly take 1 Myr to accrete the amount of mass already present in the Musca filament. Based on the estimated mass accretion 
rates and time scales, we find that strands can be provided with sufficient mass from the more extend ambient cloud during the timescale of their accretion on the filament crest. This allows for a continuous mass accretion on the Musca filament.

Several theoretical studies have investigated the collapse timescales of non spherical structures (e.g. Burkert \& Hartmann 2004; Toalá et al. 2012; Pon et al. 2012; Clarke \& Whitworth 2015). To estimate the longitudinal collapse timescale of the Musca filament, we use the formula from Clarke \& Whitworth (2015):

$t_{\text {coll }}=\left(0.49+0.26 A_{0}\right)\left(G n_{\mathrm{H}_{2}}\right)^{-\frac{1}{2}}$,

where $A_{0}$ is the initial aspect ratio, which is 54 using a half length of $3 \mathrm{pc}$ and a radius of $0.056 \mathrm{pc}$ for Musca (Cox et al. 2016, corrected to $d=140 \mathrm{pc}), G$ is the gravitational constant, and for $n_{\mathrm{H}_{2}}$, the molecular hydrogen gas density, we use $10^{4} \mathrm{~cm}^{-3}$. This results in a timescale of $9.2 \mathrm{Myr}$, which is roughly an order of magnitude larger than the estimated accretion time scale for the large scale inflowing mass reservoir. This lifetime for longitudinal collapse is a lower limit because of possible magnetic field support.

We thus find a scenario where the Musca filament crest is a long-lived filament because it is a coherent structure with sufficient continuous mass accretion which is driven by the bending of the magnetic field due to the large scale colliding $\mathrm{H}_{\text {I }}$ flow that forms the Chamaeleon-Musca complex. As this provides a sufficient amount of mass for Musca to become supercritical before it is dispersed, gravity starts playing a more important role in further confining the filament. The future continuous mass accretion, which might be increasingly driven by gravity, could further increase the density in the filament. This can lead to further fragmentation of the Musca filament, which has started in several regions of the filament (Kainulainen et al. 2016), to form pre-stellar and protostellar cores in a dynamic filamentary structure.

\section{Conclusion}

We have presented APEX observations of $\mathrm{CO}(2-1)$ isotopologues towards the Musca filament crest and the strands. This data was complemented with NANTEN2 observations of ${ }^{12} \mathrm{CO}(1-0)$ covering the full Musca cloud. We find that $\mathrm{C}^{18} \mathrm{O}$ traces the filament crest, ${ }^{13} \mathrm{CO}$ the strands and ${ }^{12} \mathrm{CO}$ the more diffuse ambient molecular cloud. The main results of this study can be summarised as follows:

- Gaia star reddening data suggests a distance of $140 \mathrm{pc}$ for Musca, but we note that there might already be some reddening at $\sim 100 \mathrm{pc}$.

- Modelling the CO lines with non-LTE line radiative transfer favours a scenario where Musca crest is a compact filament with a central density $n_{\mathrm{H}_{2}} \sim 10^{4} \mathrm{~cm}^{-3}$ at $T_{\mathrm{K}} \sim 10 \mathrm{~K}$, and where the strands are an independent feature, consisting of dense gas (with $n_{\mathrm{H}_{2}} \sim 10^{3}-3 \times 10^{3} \mathrm{~cm}^{-3}$ at $T_{\mathrm{K}} \sim 15 \mathrm{~K}$ ) that is connected to the filament crest.

- We report a sharp increase of the $\left[{ }^{13} \mathrm{CO}\right] /\left[\mathrm{C}^{18} \mathrm{O}\right]$ abundance ratio by roughly an order of magnitude over a small distance $(<0.2 \mathrm{pc})$ at $A_{\mathrm{V}}<3$. This occurs in a weak ambient FUV field $\left(<1 \mathrm{G}_{0}\right)$ and indicates $\mathrm{C}^{18} \mathrm{O}$ is a limited column density tracer.

- We confirm that the filament crest is a velocity-coherent structure, and also demonstrate that there are transverse velocity gradients over the velocity-coherent filament crest with a magnitude similar to the transonic linewidth of the filament crest.
- The ambient cloud contains a significant amount of blueshifted gas along the line of sight with respect to the filament crest, while there is a lack of redshifted gas with respect to the filament crest.

- We observe a link between the transverse velocity gradient over the filament crest and the location of the blueshifted strands, indicating that the strands are accreted on the filament crest and that the velocity gradient is a signature of this accretion which possibly also deposits some angular momentum in the crest.

- We find a kinematically coherent asymmetry from the $\sim 50 \mathrm{pc} \mathrm{Hi}$ cloud down to the Musca filament crest as well as an asymmetric column density profile for the Musca cloud, indicating that the large scale evolution of the Chamaeleon-Musca complex is directly related to the formation of the Musca filament and that the asymmetry contains essential information on the physical process responsible for dense gas formation in the region.

- The PV diagram of the Musca cloud traces a V-shape with the filament crest located at the redshifted apex of this V-shape.

Combining all data, we propose that the Musca filament crest is a long-lived dynamic filament because it is a coherent structure that is continuously replenished by inflowing gas. This mass accretion is driven by the colliding $\mathrm{H}_{\mathrm{I}}$ flow that forms the Chamaeleon-Musca complex and can eventually lead to the formation of protostellar cores embedded in this dynamic filament. This colliding flow in Chamaeleon-Musca is the result of a magnetised low-velocity H i cloud-cloud collision that produces the observed asymmetric accretion scenario, seen as a V-shape in the PV diagram, driven by the bending of the magnetic field.

Acknowledgements. The authors thank the anonymous referee for providing insightful input that improved the clarity and quality of this paper. L.B., N.S., R.S., and S.B. acknowledge support by the french ANR and the german DFG through the project "GENESIS" (ANR-16-CE92-0035-01/DFG1591/2-1). L.B. also acknowledges support from the Région Nouvelle-Aquitaine. N.S. acknowledges support from the BMBF, Projekt Number 50OR1714 (MOBSMOdellierung von Beobachtungsdaten SOFIA). N.S. and R.S. are supported by the German Deutsche Forschungsgemeinschaft, DFG project number SFB 956. We thank F. Wyrowski for providing information to correct for the frequency shift in the APEX FLASH observations. We thank F. Boulanger for input on the production of the Planck polarisation maps. This research made use of Astropy (http: //www . astropy.org), a community-developed core Python package for Astronomy (Astropy Collaboration 2013, 2018). Based on observations obtained with Planck (http://www.esa.int/Planck), an ESA science mission with instruments and contributions directly funded by ESA Member States, NASA, and Canada. This publication is based on data acquired with the Atacama Pathfinder Experiment (APEX) under programme IDs 0100.C-0825(A), 0101.F9511(A) and 0102.F-9503(A). This research has made use of data from the Herschel Gould Belt survey project (http://gouldbelt-herschel. cea.fr). The HGBS is a Herschel Key Project jointly carried out by SPIRE Specialist Astronomy Group 3 (SAG3), scientists of several institutes in the PACS Consortium (CEA Saclay, INAF-IAPS Rome and INAF-Arcetri, KU Leuven, MPIA Heidelberg), and scientists of the Herschel Science Center (HSC).

\section{References}

Alves de Oliveira, C., Schneider, N., Merín, B., et al. 2014, A\&A, 568, A98 Andrae, R., Fouesneau, M., Creevey, O., et al. 2018, A\&A, 616 A8 André, P., Men'shchikov, A., Bontemps, S., et al. 2010, A\&A, 518, L102 André, P., Di Francesco, J., Ward-Thompson, D., et al. 2014, Protostars and Planets VI (Tucson, AZ: University of Arizona Press), 27

Arzoumanian, D., André, P., Didelon, P., et al. 2011, A\&A, 529, L6 Arzoumanian, D., Shimajiri, Y., Inutsuka, S.-i., Inoue, T., \& Tachihara, K. 2018, PASJ, 70, 96

Arzoumanian, D., André, P., Könyves, V., et al. 2019, A\&A, 621, A42 Astropy Collaboration (Robitaille, T. P., et al.) 2013, A\&A, 558, A33 Astropy Collaboration (Price-Whelan, A. M., et al. ) 2018, AJ, 156, 123 Audit, E., \& Hennebelle, P. 2005, A\&A, 433, 1 
Ballesteros-Paredes, J., Hartmann, L., \& Vázquez-Semadeni, E. 1999, ApJ, 527, 285

Banerjee, R., Vázquez-Semadeni, E., Hennebelle, P., \& Klessen, R. S. 2009, MNRAS, 398, 1082

Beuther, H., Ragan, S. E., Johnston, K., et al. 2015, A\&A, 584, A67

Bohlin, R. C., Savage, B. D., \& Drake, J. F. 1978, ApJ, 224, 132

Bonne, L., Schneider, N., Bontemps, S., et al. 2020, A\&A, 641, A17

Bontemps, S., André, P., Könyves, V., et al. 2010, A\&A, 518, L85

Braine, J., Rosolowsky, E., Gratier, P., Corbelli, E., \& Schuster, K.-F. 2018, A\&A, 612, A51

Braine, J., Hughes, A., Rosolowsky, E., et al. 2020, A\&A, 633, A17

Burkert, A., \& Hartmann, L. 2004, ApJ, 616, 288

Chen, C.-Y., \& Ostriker, E. C. 2014, ApJ, 785, 69

Chen, C.-Y., Mundy, L. G., Ostriker, E. C., Storm, S., \& Dhabal, A. 2020 MNRAS, 494, 2675

Clarke, S. D., \& Whitworth, A. P. 2015, MNRAS, 449, 1819

Clarke, S. D., Whitworth, A. P., Duarte-Cabral, A., \& Hubber, D. A. 2017, MNRAS, 468, 2489

Clarke, S. D., Whitworth, A. P., Spowage, R. L., et al. 2018, MNRAS, 479, 1722

Corradi, W. J. B., Franco, G. A. P., \& Knude, J. 1997, A\&A, 326, 1215

Cox, N. L. J., Arzoumanian, D., André, P., et al. 2016, A\&A, 590, A110

Csengeri, T., Bontemps, S., Schneider, N., Motte, F., \& Dib, S. 2011, A\&A, 527, A 135

Dhabal, A., Mundy, L. G., Rizzo, M. J., Storm, S., \& Teuben, P. 2018, ApJ, 853, 169

Duarte-Cabral, A., \& Dobbs, C. L. 2017, MNRAS, 470, 4261

Federrath, C. 2016, MNRAS, 457, 375

Franco, G. A. P. 1991, A\&A, 251, 581

Fukui, Y., Inoue, T., Hayakawa, T., \& Torii, K. 2020, PASP, in press [arXiv:1909.08202]

Gaia Collaboration (Brown, A. G. A., et al.) 2018, A\&A, 616, A1

Galli, D., Walmsley, M., \& Gonçalves J. 2002, A\&A, 394, 275

Glassgold, A. E., Huggins, P. J., \& Langer, W. D. 1985, ApJ, 290, 615

Goldsmith, P. F. 2001, ApJ, 557, 736

Goldsmith, P. F., Heyer, M., Narayanan, G., et al. 2008, ApJ, 680, 428

Gómez, G. C., \& Vázquez-Semadeni, E. 2014, ApJ, 791, 124

Güsten, R., Nyman, L. A., Schilke, P., et al. 2006, A\&A, 454, L13

Güver, T., \& Özel, F. 2009, MNRAS, 400, 2050

Hacar, A., \& Tafalla, M. 2011, A\&A, 533, A34

Hacar, A., Tafalla, M., Kauffmann, J., \& Kovács, A. 2013, A\&A, 554, A55

Hacar, A., Kainulainen, J., Tafalla, M., Beuther, H., \& Alves, J. 2016, A\&A, 587, A97

Heitsch, F. 2013, ApJ, 769, 115

Heitsch, F., Burkert, A., Hartmann, L. W., Slyz, A. D., \& Devriendt, J. E. G. 2005, ApJ, 633, L113

Hennebelle, P. 2013, A\&A, 556, A153

Hennebelle, P., \& André, P. 2013, A\&A, 560, A68

Hennebelle, P., Banerjee, R., Vázquez-Semadeni, E., Klessen, R. S., \& Audit, E 2008, A\&A, 486, L43

Hennemann, M., Motte, F., Schneider, N., et al. 2012, A\&A, 543, L3

Henshaw, J. D., Jiménez-Serra, I., Longmore, S. N., et al. 2017, MNRAS, 464, L31

Hill, T., Motte, F., Didelon, P., et al. 2011, A\&A, 533, A94

Inoue, T., \& Fukui, Y. 2013, ApJ, 774, L31

Inoue, T., \& Inutsuka, S.-i. 2012, ApJ, 759, 35

Inoue, T., Hennebelle, P., Fukui, Y., et al. 2018, PASJ, 70, S53

Inutsuka, S.-I., \& Miyama, S. M. 1992, ApJ, 388, 392

Inutsuka, S.-i., \& Miyama, S. M. 1997, ApJ, 480, 68

Ivlev, A. V., Silsbee, K., Sipilä, O., \& Caselli, P. 2019, ApJ, 884, 176

Iwasaki, K., Tomida, K., Inoue, T., \& Inutsuka, S.-i. 2019, ApJ, 873, 6

Juvela, M., Ristorcelli, I., Pagani, L., et al. 2012, A\&A, 541, A12

Kainulainen, J., Beuther, H., Henning, T., \& Plume, R. 2009, A\&A, 508, L35

Kainulainen, J., Hacar, A., Alves, J., et al. 2016, A\&A, 586, A27

Kalberla, P. M. W., \& Haud, U. 2015, A\&A, 578, A78

Kalberla, P. M. W., McClure-Griffiths, N. M., Pisano, D. J., et al. 2010, A\&A, 521, A17

Klein, T., Ciechanowicz, M., Leinz, C., et al. 2014, IEEE Trans. Terahertz Sci. Technol., 4, 588

Knude, J., \& Hog, E. 1998, A\&A, 338, 897

Koch, E. W., \& Rosolowsky, E. W. 2015, MNRAS, 452, 3435

Könyves, V., André, P., Men'shchikov, A., et al. 2010, A\&A, 518, L106

Könyves, V., André, P., Men'shchikov, A., et al. 2015, A\&A, 584, A91

Koyama, H., \& Inutsuka, S.-i. 2002, ApJ, 564, L97
Langer, W. D., \& Penzias, A. A. 1990, ApJ, 357, 477

Liszt, H., Gerin, M., \& Grenier, I. 2019, A\&A 627, A95

Loison, J.-C., Wakelam, V., Gratier, P., et al. 2019, MNRAS, 485, 5777

Machaieie, D. A., Vilas-Boas, J. W., Wuensche, C. A., et al. 2017, ApJ, 836, 19

Malinen, J., Montier, L., Montillaud, J., et al. 2016, MNRAS, 460, 1934

Marsh, K. A., Kirk, J. M., André, P., et al. 2016, MNRAS, 459, 342

McClure-Griffiths, N. M., Pisano, D. J., Calabretta, M. R., et al. 2009, ApJS, 181, 398

Merello, M., Molinari, S., Rygl, K. L. J., et al. 2019, MNRAS, 483, 5355

Mizuno, A., Hayakawa, T., Yamaguchi, N., et al. 1998, ApJ, 507, L83

Mizuno, A., Yamaguchi, R., Tachihara, K., et al. 2001, PASJ, 53, 1071

Moeckel, N., \& Burkert, A. 2015, ApJ, 807, 67

Molinari, S., Swinyard, B., Bally, J., et al. 2010, A\&A, 518, L100

Motte, F., Bontemps, S., \& Louvet, F. 2018, ARA\&A, 56, 41

Myers, P. C. 2009, ApJ, 700, 1609

Nagai, T., Inutsuka, S.-i., \& Miyama, S. M. 1998, ApJ, 506, 306

Nakamura, F., \& Li, Z.-Y. 2008, ApJ, 687, 354

Nielbock, M., Launhardt, R., Steinacker, J., et al. 2012, A\&A, 547, A11

Ossenkopf-Okada, V., \& Stepanov, R. 2019, A\&A, 621, A5

Ostriker, J. 1964, ApJ, 140, 1056

Padoan, P., Juvela, M., Goodman, A. A., \& Nordlund, Å. 2001, ApJ, 553, 227

Palmeirim, P., André, P., Kirk, J., et al. 2013, A\&A, 550, A38

Panopoulou, G. V., Psaradaki, I., Skalidis, R., Tassis, K., \& Andrews, J. J. 2017, MNRAS, 466, 2529

Peretto, N., André, P., Könyves, V., et al. 2012, A\&A, 541, A63

Peretto, N., Fuller, G. A., Duarte-Cabral, A., et al. 2013, A\&A, 555, A112

Peretto, N., Fuller, G. A., André, P., et al. 2014, A\&A, 561, A83

Pereyra, A., \& Magalhães, A. M. 2004, ApJ, 603, 584

Pilbratt, G. L., Riedinger, J. R., Passvogel, T., et al. 2010, A\&A, 518, L1

Planck Collaboration I. 2016, A\&A, 594, A1

Planck Collaboration Int. XXVIII. 2015, A\&A, 582, A31

Planck Collaboration Int. XIII. 2016, A\&A, 586, A136

Planck Collaboration Int. XXXV. 2016, A\&A, 586, A138

Polychroni, D., Schisano, E., Elia, D., et al. 2013, ApJ, 777, L33

Pon, A., Toalá, J. A., Johnstone, D., et al. 2012, ApJ, 756, 145

Rachford, B. L., Snow, T. P., Destree, J. D., et al. 2009, ApJS, 180, 125

Recchi, S., Hacar, A., \& Palestini, A. 2014, MNRAS, 444, 1775

Röllig, M., \& Ossenkopf, V. 2013, A\&A, 550, A56

Roy, A., André, P., Palmeirim, P., et al. 2014, A\&A, 562, A138

Rygl, K. L. J., Benedettini, M., Schisano, E., et al. 2013, A\&A, 549, L1

Schneider, N., Csengeri, T., Bontemps, S., et al. 2010, A\&A, 520, A49

Schneider, N., Bontemps, S., Simon, R., et al. 2011, A\&A, 529, A1

Schneider, N., Csengeri, T., Hennemann, M., et al. 2012, A\&A, 540, L11

Schöier, F. L., van der Tak, F. F. S., van Dishoeck, E. F., \& Black, J. H. 2005, A\&A, 432, 369

Seifried, D., \& Walch, S. 2015, MNRAS, 452, 2410

Seifried, D., \& Walch, S. 2016, MNRAS, 459, L11

Seifried, D., Sánchez-Monge, Á., Suri, S., \& Walch, S. 2017, MNRAS, 467, 4467

Shimajiri, Y., André, P., Palmeirim, P., et al. 2019, A\&A, 623, A16

Smith, R. J., Glover, S. C. O., \& Klessen, R. S. 2014, MNRAS, 445, 2900

Smith, R. J., Glover, S. C. O., Klessen, R. S., \& Fuller, G. A. 2016, MNRAS, 455,3640

Soler, J. D., Hennebelle, P., Martin, P. G., et al. 2013, ApJ, 774, 128

Szúcs, L., Glover, S. C. O., \& Klessen, R. S. 2014, MNRAS, 445, 4055

Tafalla, M., \& Hacar, A. 2015, A\&A, 574, A104

Toalá, J. A., Vázquez-Semadeni, E., \& Gómez, G. C. 2012, ApJ, 744, 190

Tritsis, A., \& Tassis, K. 2016, MNRAS, 462, 3602

Tritsis, A., \& Tassis, K. 2018, Science, 360, 635

Vaidya, B., Hartquist, T. W., \& Falle, S. A. E. G. 2013, MNRAS, 433, 1258

Vallée, J. P., \& Fiege, J. D. 2006, ApJ, 636, 332

van der Tak, F. F. S., Black, J. H., Schöier, F. L., Jansen, D. J., \& van Dishoeck, E. F. 2007, A\&A, 468, 627

van Dishoeck, E. F., \& Black, J. H. 1988, ApJ, 334, 771

Vilas-Boas, J. W. S., Myers, P. C., \& Fuller, G. A. 1994, ApJ, 433, 96

Visser, R., van Dishoeck, E. F., \& Black, J. H. 2009, A\&A, 503, 323

Whittet, D. C. B., Prusti, T., Franco, G. A. P., et al. 1997, A\&A, 327, 1194

Williams, G. M., Peretto, N., Avison, A., Duarte-Cabral, A., \& Fuller, G. A. 2018, A\&A, 613, A11

Yan, Q.-Z., Zhang, B., Xu, Y., et al. 2019, A\&A, 624, A6

Zamora-Avilés, M., Ballesteros-Paredes, J., \& Hartmann, L. W. 2017, MNRAS, 472, 647

Zielinsky, M., Stutzki, J., \& Störzer, H. 2000, A\&A, 358, 723

Zucker, C., Speagle, J. S., Schlafly, E. F., et al. 2019, ApJ, 879, 125 


\section{Appendix A: Distance of the dense Musca-Chamaeleon clouds}
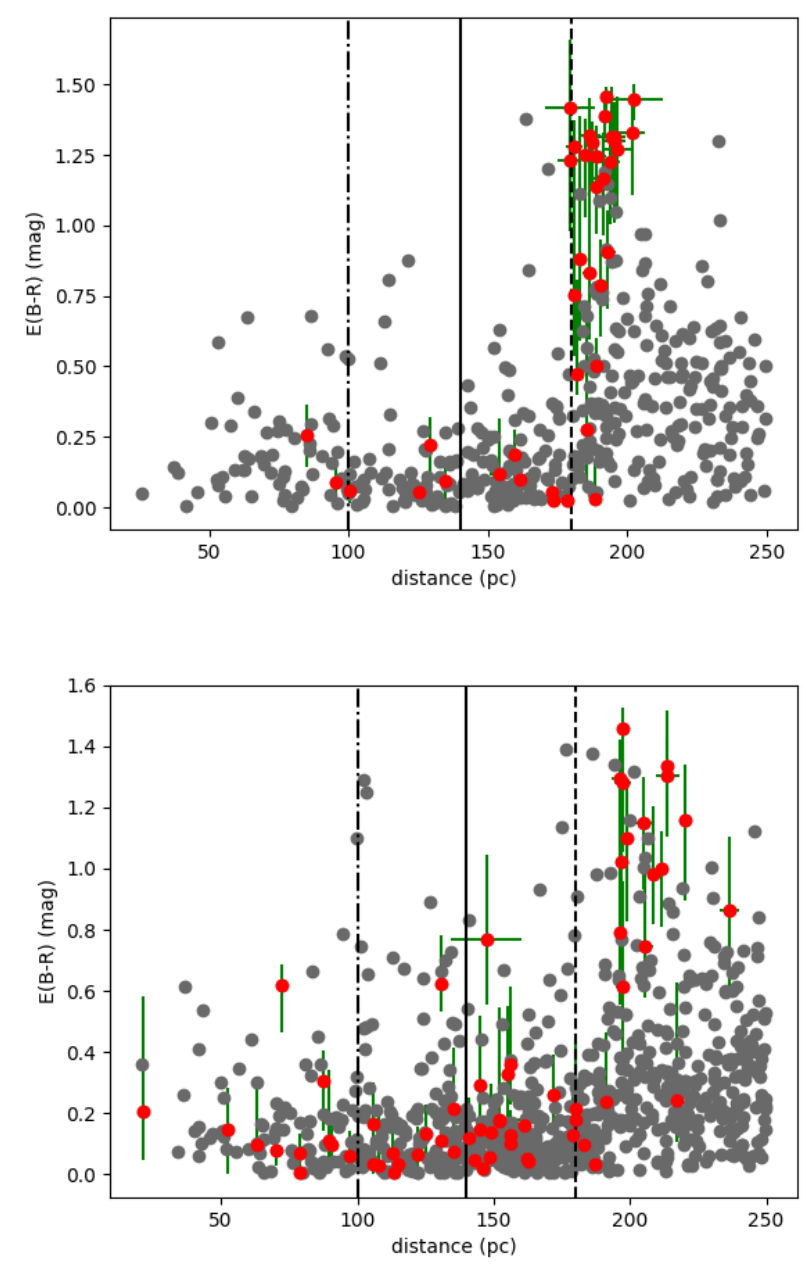

Fig. A.1. Top: reddening as a function of distance for stars in the area covered by Cha I. As in Fig. 5, the red points are located at the high density region of the cloud. This shows that the reddening experiences a strong jump around $180 \mathrm{pc}$ as was found in Zucker et al. (2019). The other two vertical lines in the plots indicate a distance of 100 and $140 \mathrm{pc}$. Bottom: same for the region Cha II and Cha III together, showing a reddening jump at $190 \mathrm{pc}$.

With the recent Gaia DR2 (Gaia Collaboration 2018) data release, a vast amount of information on nearby stars in the Galaxy has become available. This makes it possible to estimate the distance of interstellar clouds by studying the reddening they cause on background stars of these clouds (e.g. Zucker et al. 2019; Yan et al. 2019). To estimate the distance of Musca, a region in the sky centered on the Musca filament with a radius of $80^{\prime}(\sim 3 \mathrm{pc}$ at a distance of $150 \mathrm{pc}$ ) was selected in the Gaia catalogue, see Fig. 1. In this region, all stars with a calculated reddening (Andrae et al. 2018) and a distance $<250 \mathrm{pc}$ were selected. This results in a total of 223 stars found that can be used to estimate the distance of Musca. Because there can be foreground reddening we only select the stars that are located in the Planck $353 \mathrm{GHz}$ map above a threshold (Yan et al. 2019).
Inspecting the Planck map by eye, a threshold of $0.006 \mathrm{~K}_{\mathrm{cmb}}$ was used. The idea behind the method is that the presence of the Musca cloud should give rise to a jump in reddening at a certain distance. The plot of the reddening as a function of the distance shows indication of such a jump at a distance of $140 \mathrm{pc}$, suggesting the presence of the Musca cloud. However, it can be noted that there already is reddening for a few stars before $140 \mathrm{pc}$. This generally starts at a distance of 90-100 pc and might indicate the presence of some gas that is more nearby than the Musca filament/cloud. Note that there generally is significant uncertainty about the reddening calculated in the Gaia catalogue (Andrae et al. 2018), such that this reddening effect before $140 \mathrm{pc}$ might appear larger than it actually is. Figure 5 shows that the nearby stars $(<140 \mathrm{pc})$ with high reddening are located all over the map. This could imply that this is not the result of nearby gas concentrated in a small region, but that such nearby gas might be present over a larger area which could be related to the ChamaeleonMusca complex. The same approach was used for Cha I, Cha II and Cha III, suggesting a distance of 180-190 pc, see Fig. A.1, which is consistent with the results from Zucker et al. (2019).

\section{Appendix B: The ${ }^{13} \mathrm{CO}(3-2) /{ }^{13} \mathrm{CO}(2-1)$ uncertainty}

To estimate the average density along the line of sight in the Musca filament and make an evolution versus distance from the filament crest, we have studied the ratio of ${ }^{13} \mathrm{CO}(3-2) /{ }^{13} \mathrm{CO}(2-$ 1). Here, we check the dependence of the density estimate on the width of the line and the column density of the line.

To study the impact of a change in width, the same setup as in Sect. 5.1 was run, but this time with a FWHM of $0.5 \mathrm{~km} \mathrm{~s}^{-1}$ (which is the minimal FWHM over the entire map). To study the impact of the column density, the setup from Sect. 5.1 was retaken but this time with $N_{\mathrm{H}_{2}}=4 \times 10^{21} \mathrm{~cm}^{-2}$ (or $33 \%$ less than the maximal column density used in Sect. 5.1). The impact of these changes are plotted in Fig. B.1. When one has a fixed ${ }^{13} \mathrm{CO}(3-2) /{ }^{13} \mathrm{CO}(2-1)$ ratio, for a lower column density this would result in a slightly higher density of the gas. The opposite is true for the FWHM: when the FWHM is lower for a fixed ${ }^{13} \mathrm{CO}(3-2) /{ }^{13} \mathrm{CO}(2-1)$ ratio, the density for this ratio will be slightly lower than for a higher FWHM. When comparing the variation of the ratio as a function of density for a different FWHM or column density with the variation related to a different temperature in Fig. B.1, it is found that the variation related to the temperature is larger than the variation related to the FWHM or column density. Furthermore, the FWHM and column density values used in Sect. 5.1 are both relatively high for the strands located around the filament such that for both parameters a lower value might be more accurate. But the above analysis indicates that the effect of lowering the FWHM and column density at the same time might roughly cancel out due to their opposite impact on the ratio. The estimated densities in the strands might thus be reasonable as well. This is confirmed when using the obtained estimates for the density in the strands and filament from the above model to characterise the gas in these components. The intensity of the observed lines towards Musca are reproduced for temperatures and column densities that can be expected from Herschel. This provides further confidence that the density estimates might be relatively good. 
L. Bonne et al.: Formation of the Musca filament: evidence for asymmetries in the accretion flow due to a cloud-cloud collision
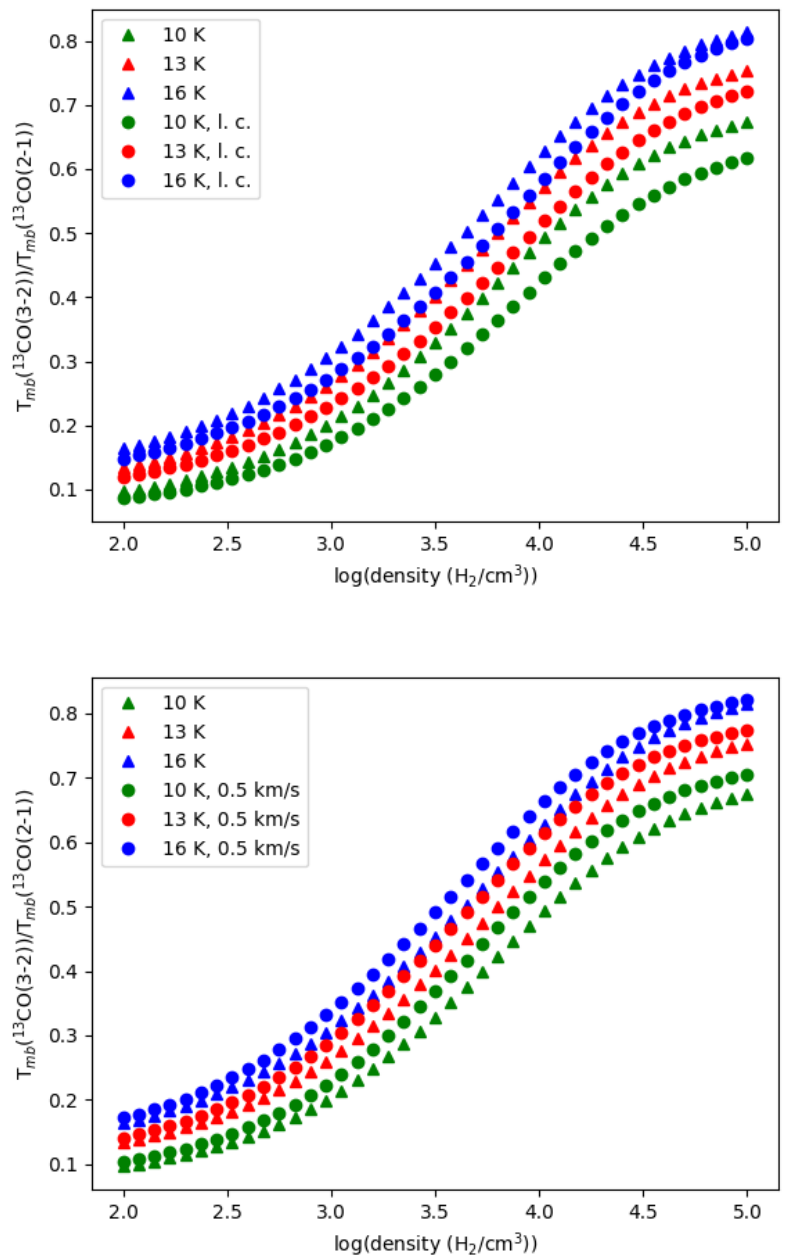

Fig. B.1. Top: impact of a lower column density, namely $N_{\mathrm{H}_{2}}=$ $4 \times 10^{21} \mathrm{~cm}^{-2}$ (indicated with 1. c.) instead of $N_{\mathrm{H}_{2}}=6 \times 10^{21} \mathrm{~cm}^{-2}$, on the ${ }^{13} \mathrm{CO}(3-2) /{ }^{13} \mathrm{CO}(2-1)$ ratio as a function the density. Bottom: impact of a lower FWHM $\left(0.5 \mathrm{~km} \mathrm{~s}^{-1}\right.$ instead of $\left.0.7 \mathrm{~km} \mathrm{~s}^{-1}\right)$ on the ${ }^{13} \mathrm{CO}(3-$ $2) /{ }^{13} \mathrm{CO}(2-1)$ ratios as a function of the density. For both the width and the column density, their impact is plotted for 3 different temperatures: 10,13 and $16 \mathrm{~K}$. This shows that the impact of these two variables is smaller than the uncertainty related to the temperature. 